\title{
THE CLUSTER INDEX OF REGULARLY VARYING SEQUENCES WITH APPLICATIONS TO LIMIT THEORY FOR FUNCTIONS OF MULTIVARIATE MARKOV CHAINS
}

\author{
THOMAS MIKOSCH AND OLIVIER WINTENBERGER
}

\begin{abstract}
We introduce the cluster index of a multivariate regularly varying stationary sequence and characterize the index in terms of the spectral tail process. This index plays a major role in limit theory for partial sums of regularly varying sequences. We illustrate the use of the cluster index by characterizing infinite variance stable limit distributions and precise large deviation results for sums of multivariate functions acting on a stationary Markov chain under a drift condition.
\end{abstract}

AMS 2000 subject classifications: Primary 60J05; Secondary 60F10, 60F05, 60G70

Keywords and phrases: Markov processes, regular variation, central limit theorem, large deviation principle, GARCH.

\section{INTRODUCTION}

Consider a stationary Markov chain $\left(\Phi_{t}\right)$ and a function $f$ acting on the state space of the Markov chain and mapping into $\mathbb{R}^{d}$ for some $d \geqslant 1$. For the resulting stationary process $X_{t}=f\left(\Phi_{t}\right), t \in \mathbb{Z}$, the corresponding partial sum process is given by

$$
S_{0}=0, \quad S_{n}=X_{1}+\cdots+X_{n} .
$$

We also assume that the finite-dimensional distributions of the process $\left(X_{t}\right)$ are regularly varying with index $\alpha$; see Section 2.1 for a definition. Roughly speaking, this condition ensures that the tails of the finite-dimensional distributions have power law behavior, hence sufficiently high moments of $X$ are infinite. (Here and in what follows, we write $Y$ for a generic element of any stationary sequence $\left(Y_{t}\right)$.) Regular variation of a random vector and, more generally, of a stationary sequence is a condition which determines the extremal dependence structure in a flexible way.

For an iid sequence the condition of regular variation of $X$ with index $\alpha \in(0,2)$ is necessary and sufficient for the central limit theorem

$$
a_{n}^{-1}\left(S_{n}-b_{n}\right) \stackrel{d}{\rightarrow} \xi_{\alpha}, \quad n \rightarrow \infty,
$$

where $a_{n}>0, b_{n} \in \mathbb{R}, n \in \mathbb{N}$, are suitable constants and $\xi_{\alpha}$ has an $\alpha$-stable distribution in $\mathbb{R}^{d}$; see 49 for the limit theorem and [50 for a description of infinite variance stable laws in $\mathbb{R}^{d}$. Limit theory with $\alpha$-stable limits for dependent sequences was studied in 28, 29] by using the convergence of characteristic functions and in 16 by using the continuous mapping theorem acting on suitable weakly converging point processes; see also [5] for a functional central limit theorem using the same technique. These results were proved for univariate sequences, but [17] proved that the point process convergence results remain valid in the multivariate case by a slight modification of the proofs in [16.

Thomas Mikosch's research is partly supported by the Danish Research Council (FNU) Grants 272-06-0442 and 09-072331. Both authors would like to thank their home institutions for hospitality when visiting each other. 
Using ideas from [28, 29, the authors of [2] studied stable limit theory for general univariate regularly varying sequences; see Theorem 6.1 below. We use this result and the Cramér-Wold device to derive the corresponding limits for all linear combinations $\theta^{\prime} S_{n}, \theta \in \mathbb{S}^{d-1}$, where $\mathbb{S}^{d-1}$ is the unit sphere in $\mathbb{R}^{d}$ with respect to the Euclidean norm. According to Theorem 6.1, the $\alpha$-stable limit laws of $\theta^{\prime} S_{n}$ (with suitable normalization and centering) are characterized by the function

$$
b(\theta)=\lim _{k \rightarrow \infty} \lim _{x \rightarrow \infty} \frac{\mathbb{P}\left(\theta^{\prime} S_{k}>x\right)-\mathbb{P}\left(\theta^{\prime} S_{k-1}>x\right)}{\mathbb{P}(|X|>x)}, \quad \theta \in \mathbb{S}^{d-1} .
$$

We discuss the so-called cluster index $b$ in Section 3. The existence of the limits in (1.1) is guaranteed under the conditions of this paper; see Theorem 3.2 Moreover, the cluster index $b$ determines the $\alpha$-stable limit laws in the multivariate case; see Theorem 4.1 In a way, the function $b$ plays a similar role as the notion of extremal index in limit theory for maxima of dependent sequences; see [35] for this notion.

Regular variation is also the key to precise large deviation theory for the sums $S_{n}$. In the univariate iid case, classical work by A.V. and S.V. Nagaev 41, 42 shows that relations of the following type hold

$$
\sup _{x \geqslant b_{n}}\left|\frac{\mathbb{P}\left(S_{n}>x\right)}{n \mathbb{P}(|X|>x)}-p\right| \rightarrow 0
$$

where $p=\lim _{x \rightarrow \infty} \mathbb{P}(X>x) / \mathbb{P}(|X|>x)=p$ and $\left(b_{n}\right)$ is a suitably chosen sequence such that $b_{n} \rightarrow \infty$ and $S_{n} / b_{n} \stackrel{P}{\rightarrow} 0$ as $n \rightarrow \infty$. Related work for dependent regularly varying sequences was proved in [39] for linear processes, in [14, 34 for solutions to stochastic recurrence equations and for general regularly varying sequences in [40; for earlier results see also [28, 29, 16]. The results in these papers are all of the type

$$
\sup _{x \in\left(b_{n}, c_{n}\right)}\left|\frac{\mathbb{P}\left(S_{n}>x\right)}{n \mathbb{P}(|X|>x)}-b(1)\right| \rightarrow 0,
$$

where $b(1)$ is the limit in (1.1) for $d=1$ and $\left(b_{n}, c_{n}\right)$ are suitable regions tending to infinity.

The case of iid multivariate regularly varying $\left(X_{t}\right)$ was treated in [27, including a corresponding functional large deviation result. In this paper, we get a corresponding large deviation principle for regularly varying multivariate functions acting on a Markov chain (see Theorem 4.3):

$$
\frac{\mathbb{P}\left(\lambda_{n}^{-1} S_{n} \in \cdot\right)}{n \mathbb{P}\left(|X|>\lambda_{n}\right)} \stackrel{v}{\rightarrow} \nu_{\alpha}
$$

Here $\stackrel{v}{\rightarrow}$ denotes vague convergence on some Borel $\sigma$-field, $\lambda_{n} \rightarrow \infty$ is a suitable normalizing sequence and the limit $\nu_{\alpha}$ is a measure which is induced by the regular variation of the sums $S_{k}, k \geqslant 1$. As for the case of stable limits, we start by proving the large deviation principle for linear combinations $\theta^{\prime} S_{n}$, exploiting the corresponding result (1.2) with $b(1)$ replaced by $b(\theta)$ from (1.1); see Theorem 7.2 This corresponds to (1.3) restricted to half-planes not containing the origin. It is in general not possible to extend the limit relation (1.3) from half-spaces to general Borel sets. This extension is however possible by assuming some additional conditions such as $\alpha$ is non-integer. As a matter of fact, relation (1.3) cannot be written as a uniform result in the spirit of (1.2), due to its multivariate character.

The paper is organized as follows. In Section 2 we introduce regular variation of a stationary sequence and the drift condition of a Markov chain. In Section 3 we define the cluster index $b(\theta)$, $\theta \in \mathbb{S}^{d-1}$, of a regularly varying stationary sequence. We prove the existence of the cluster index for multivariate functions acting on a Markov chain under a drift condition (Theorem 3.2). In Section 4 we formulate the main results of this paper. They include $\alpha$-stable limit theory (Theorem 4.1) and precise large deviation principles (Theorem 4.2) for functions of regenerative Markov chains. 
In Section [5 we calculate the cluster index for several important time series models, including multivariate autoregressive processes, solutions to stochastic recurrence equations, $\operatorname{GARCH}(1,1)$ processes and their sample covariance functions. In the remaining sections we prove the results of Section 4

\section{Preliminaries}

2.1. Regular variation of vectors and sequences of random vectors. In what follows, we will use the notion of regular variation as a suitable way of describing heavy tails of random vectors and sequences of random vectors. We commence with a random vector $X$ with values in $\mathbb{R}^{d}$ for some $d \geqslant 1$. We say that this vector (and its distribution) are regularly varying with index $\alpha>0$ if the following relation holds as $x \rightarrow \infty$ :

$$
\frac{\mathbb{P}(|X|>u x, X /|X| \in \cdot)}{\mathbb{P}(|X|>x)} \stackrel{w}{\rightarrow} u^{-\alpha} \mathbb{P}(\Theta \in \cdot), \quad u>0 .
$$

Here $\stackrel{w}{\rightarrow}$ denotes weak convergence of finite measures and $\Theta$ is a vector with values in the unit sphere $\mathbb{S}^{d-1}=\left\{x \in \mathbb{R}^{d}:|x|=1\right\}$ of $\mathbb{R}^{d}$. Its distribution is the spectral measure of regular variation and depends on the choice of the norm. However, the definition of regular variation does not depend on any concrete norm; we always refer to the Euclidean norm. An equivalent way to define regular variation of $X$ is to require that there exists a non-null Radon measure $\mu$ on the Borel $\sigma$-field of $\overline{\mathbb{R}}_{0}^{d}=\overline{\mathbb{R}}^{d} \backslash\{\mathbf{0}\}$ such that

$$
n \mathbb{P}\left(a_{n}^{-1} X \in \cdot\right) \stackrel{v}{\rightarrow} \mu_{X}(\cdot),
$$

where the sequence $\left(a_{n}\right)$ can be chosen such that $n \mathbb{P}\left(|X|>a_{n}\right) \sim 1$ and $\stackrel{v}{\rightarrow}$ refers to vague convergence. The limit measure $\mu_{X}$ necessarily has the property $\mu_{X}(u \cdot)=u^{-\alpha} \mu_{X}(\cdot), u>0$, which explains the relation with the index $\alpha$. We refer to [7] for an encyclopedic treatment of one-dimensional regular variation and [4, 47, for the multivariate case.

Next consider a strictly stationary sequence $\left(X_{t}\right)_{t \in \mathbb{Z}}$ of $\mathbb{R}^{d}$-valued random vectors with a generic element $X$. It is regularly varying with index $\alpha>0$ if every lagged vector $\left(X_{1}, \ldots, X_{k}\right), k \geqslant 1$, is regularly varying in the sense of (2.1); see [16]. An equivalent description of a regularly varying sequence $\left(X_{t}\right)$ is achieved by exploiting (2.2): for every $k \geqslant 1$, there exists a non-null Radon measure $\mu_{k}$ on the Borel $\sigma$-field of $\overline{\mathbb{R}}_{0}^{d k}$ such that

$$
n \mathbb{P}\left(a_{n}^{-1}\left(X_{1}, \ldots, X_{k}\right) \in \cdot\right) \stackrel{v}{\rightarrow} \mu_{k},
$$

where $\left(a_{n}\right)$ is chosen such that $n \mathbb{P}\left(\left|X_{0}\right|>a_{n}\right) \sim 1$.

A convenient characterization of a regularly varying sequence $\left(X_{t}\right)$ was given in Theorem 2.1 of [6]: there exists a sequence of $\mathbb{R}^{d}$-valued random vectors $\left(Y_{t}\right)_{t \in \mathbb{Z}}$ such that $\mathbb{P}\left(\left|Y_{0}\right|>y\right)=y^{-\alpha}$ for $y>1$ and for $k \geqslant 0$,

$$
\mathbb{P}\left(x^{-1}\left(X_{-k}, \ldots, X_{k}\right) \in \cdot|| X_{0} \mid>x\right) \stackrel{w}{\rightarrow} \mathbb{P}\left(\left(Y_{-k}, \ldots, Y_{k}\right) \in \cdot\right), \quad x \rightarrow \infty .
$$

The process $\left(Y_{t}\right)$ is the tail process of $\left(X_{t}\right)$. Writing $\Theta_{t}=Y_{t} /\left|Y_{0}\right|$ for $t \in \mathbb{Z}$, one also has for $k \geqslant 0$,

$$
\mathbb{P}\left(\left|X_{0}\right|^{-1}\left(X_{-k}, \ldots, X_{k}\right) \in \cdot|| X_{0} \mid>x\right) \stackrel{w}{\rightarrow} \mathbb{P}\left(\left(\Theta_{-k}, \ldots, \Theta_{k}\right) \in \cdot\right), \quad x \rightarrow \infty .
$$

We will identify $\left|Y_{0}\right|\left(Y_{t} /\left|Y_{0}\right|\right)_{|t| \leqslant k}=\left|Y_{0}\right|\left(\Theta_{t}\right)_{|t| \leqslant k}, k \geqslant 0$. Then $\left|Y_{0}\right|$ is independent of $\left(\Theta_{t}\right)_{|t| \leqslant k}$ for every $k \geqslant 0$. We refer to $\left(\Theta_{t}\right)_{t \in \mathbb{Z}}$ as the spectral tail process of $\left(X_{t}\right)$.

We formulate our main condition on the tails of the sequence $\left(X_{t}\right)$ :

Condition $\left(\mathbf{R V}_{\alpha}\right)$ : The strictly stationary sequence $\left(X_{t}\right)$ is regularly varying with index $\alpha>0$ and spectral tail process $\left(\Theta_{t}\right)$. 
2.2. The drift condition. Assume that the following drift condition holds for the Markov chain $\left(\Phi_{t}\right)$ for suitable $p>0$ and an $\mathbb{R}^{d}$-valued function $f$ acting on the state space of the Markov chain: Condition $\left(\mathbf{D C}_{p}\right)$ : There exist constants $\beta \in(0,1), b>0$, and a function $V: \mathbb{R}^{d} \rightarrow(0, \infty)$ such that $c_{1}|x|^{p} \leqslant V(x) \leqslant c_{2}|x|^{p}, c_{1}, c_{2}>0$, satisfying for any $y$ in the state space of the Markov chain,

$$
\mathbb{E}\left(V\left(f\left(\Phi_{1}\right)\right) \mid \Phi_{0}=y\right) \leqslant \beta V(f(y))+b .
$$

We mention that Jensen's inequality ensures that $\left(\mathbf{D C}_{p}\right)$ implies $\left(\mathbf{D C}_{p^{\prime}}\right)$ for $p^{\prime}<p$. We exploited condition $\left(\mathbf{D C}_{p}\right)$ in [40, where we proved large deviation principles for regularly varying strictly stationary sequences of random variables, in particular for irreducible Markov chains.

If $\left(\Phi_{t}\right)$ is an irreducible Markov chain then $\left(\mathbf{D C}_{p}\right)$ for any $p>0$ implies $\beta$-mixing with geometric rate; see [36], p. 371. Moreover, without loss of generality, by considering the Nummelin splitting scheme, see 43 for details, we will assume that $\left(\Phi_{t}\right)$ possesses an atom $A$. The notions of drift, small set, atom, etc. used throughout are borrowed from [36. In what follows, we write $\mathbb{P}_{A}(\cdot)=$ $\mathbb{P}\left(\cdot \mid \Phi_{0} \in A\right)$ and $\mathbb{E}_{A}$ for the corresponding expectation.

We always assume the existence of some $M>0$ such that $\{x: V(f(x)) \leqslant M\}$ is a small set (this is true in all our examples). Then the condition $\left(\mathbf{D C}_{p}\right)$ is equivalent to the existence of constants $\beta \in(0,1)$ and $b>0$ such that for any $y$,

$$
\mathbb{E}\left(V\left(f\left(\Phi_{1}\right) \mid \Phi_{0}=y\right) \leqslant \beta V(f(y))+b \mathbb{1}_{A}(y) .\right.
$$

Direct verification of the condition $\left(\mathbf{D C}_{p}\right)$ is in general difficult. We will use the following result which can often be checked much easier.

Lemma 2.1. Assume that the stationary Markov chain $\left(\Phi_{t}\right)$ is aperiodic, irreducible and satisfies the following condition for some $p>0$ and integer $m \geqslant 1$ :

Condition $\left(\mathbf{D C}_{p, m}\right)$ : (a) There exist $b>0$ and $\beta \in(0,1)$ such that for any $y$ in the state space of the Markov chain,

$$
\mathbb{E}\left(V\left(f\left(\Phi_{m}\right)\right) \mid \Phi_{0}=y\right) \leqslant \beta V(f(y))+b \mathbb{1}_{A}(y),
$$

where $V$ is the function from $\left(\mathbf{D C}_{p}\right)$.

(b) There exist $c_{1}, c_{2}>0$ such that for any $y$ in the state space of the Markov chain

$$
\mathbb{E}\left(V\left(f\left(\Phi_{1}\right) \mid \Phi_{0}=y\right) \leqslant c_{1} V(f(y))+c_{2} .\right.
$$

Then condition $\left(\mathbf{D C}_{p}\right)$ holds.

Proof. Theorem 15.3.3 in [36] says that the drift condition in part (a) of $\left(\mathbf{D C}_{p, m}\right)$ implies $V$ geometric regularity of the $m$-skeleton Markov chain $\left(\Phi_{t m}\right)$. Theorem 15.3.6 in [36] yields the equivalence between $V$-geometric regularity and $g$-geometric regularity of the original Markov chain for a function $g$ satisfying $\sum_{t=1}^{m} \mathbb{E}\left(g\left(\Phi_{t}\right) \mid \Phi_{0}=y\right)=V(f(y))$. Thus the drift condition is satisfied for the original Markov chain and some finite Lyapunov function $V^{\prime} \geqslant g$. Making multiple use of part (b) of $\left(\mathbf{D C}_{p, m}\right)$, we can show that there exist constants $c_{1}^{\prime}, c_{2}^{\prime}>0$ satisfying $\sum_{t=1}^{m} \mathbb{E}\left(g\left(\Phi_{t}\right) \mid\right.$ $\left.\Phi_{0}=y\right) \leqslant c_{1}^{\prime} V(f(y))+c_{2}^{\prime}$. Thus $\left(\mathbf{D C}_{p}\right.$ ) follows for a function $V^{\prime}(x)=c_{1}^{\prime \prime} V(x)+c_{2}^{\prime \prime}$ and suitable constants $c_{1}^{\prime \prime}, c_{2}^{\prime \prime}>0$.

Consider the sequence of the hitting times of the atom $A$ by the Markov chain $\left(\Phi_{t}\right)$, i.e. $\tau_{A}(1)=$ $\tau_{A}=\min \left\{k>0: \Phi_{k} \in A\right\}$ and $\tau_{A}(j+1)=\min \left\{k>\tau_{A}(j): \Phi_{k} \in A\right\}, j \geqslant 1$. We will write

$$
S(0)=\sum_{t=1}^{\tau_{A}} X_{t} \quad \text { and } \quad S(i)=\sum_{t=\tau_{A}(i)+1}^{\tau_{A}(i+1)} X_{t}, \quad i \geqslant 1 .
$$


THE CLUSTER INDEX

According to the theory in [36], $\left(\tau_{A}(i)-\tau_{A}(i-1)\right)_{i \geqslant 2}$ and $(S(i))_{i \geqslant 1}$ constitute iid sequences; we will refer to regenerative Markov chains. The drift condition $\left(\mathbf{D C}_{p}\right)$ is tailored for proving the existence of moments of $S(1)$ under the existence of moments of $X_{t}=f\left(\Phi_{t}\right)$ of the same order.

The drift condition $\left(\mathbf{D C}_{p}\right)$ is useful for proving central limit theory and other asymptotic results for functions of Markov chains. As a benchmark result we quote a central limit theorem which is a simple corollary of Proposition 2.1 in Samur [51. To apply this result notice that $\left(\mathbf{D C}_{1}\right)$ implies condition $\left(\mathrm{D}_{2}\right)$ of $\left[51\right.$ for $\left|X_{t}\right|$ with $V=c|f|$ with $c>0$ sufficiently small.

Theorem 2.2. Assume that the stationary Markov chain $\left(\Phi_{t}\right)$ is aperiodic, irreducible and $\left(X_{t}\right)=$ $\left(f\left(\Phi_{t}\right)\right)$ satisfies $\left(\mathbf{D C}_{1}\right), \mathbb{E}|X|^{2}<\infty$ and $\mathbb{E} X=0$. Then the following statements hold:

(1) The partial sum $S(1)$ has finite second moment.

(2) The central limit theorem $n^{-0.5} S_{n} \stackrel{d}{\rightarrow} \mathcal{N}(0, \Sigma)$ holds with

$$
\begin{aligned}
\Sigma & =\mathbb{E}_{A}\left[S(1) S(1)^{\prime}\right] \\
& =\lim _{k \rightarrow \infty} \mathbb{E}\left[\left(\sum_{t=0}^{k} X_{t}\right)\left(\sum_{t=0}^{k} X_{t}\right)^{\prime}-\left(\sum_{t=1}^{k} X_{t}\right)\left(\sum_{t=1}^{k} X_{t}\right)^{\prime}\right] .
\end{aligned}
$$

Together with Theorem 4.1 that deals with the case of infinite variance stable limits, Theorem 2.2 complements the limit theory for partial sums of functions of Markov chains in the case of finite variance summands and Gaussian limits.

\section{The Cluster INDEX}

We commence by considering a general $\mathbb{R}^{d}$-valued stationary process $\left(X_{t}\right)$ satisfying $\left(\mathbf{R V}_{\alpha}\right)$ for some $\alpha>0$. A continuous mapping argument for regular variation (see e.g. 24, 25]) and (2.3) ensure the existence of the limits

$$
b_{k}(\theta)=\lim _{n \rightarrow \infty} n \mathbb{P}\left(\theta^{\prime} S_{k}>a_{n}\right), \quad k \geqslant 1, \quad \theta \in \mathbb{S}^{d-1} .
$$

The difference $b_{k+1}(\theta)-b_{k}(\theta)$ can be expressed in terms of the spectral tail process $\left(\Theta_{t}\right)$ of $\left(X_{t}\right)$.

Lemma 3.1. Let $\left(X_{t}\right)$ be an $\mathbb{R}^{d}$-valued stationary process satisfying $\left(\mathbf{R V}_{\alpha}\right)$ for some $\alpha>0$. Then, for any $k \geqslant 1$,

$$
b_{k+1}(\theta)-b_{k}(\theta)=\mathbb{E}\left[\left(\theta^{\prime} \sum_{t=0}^{k} \Theta_{t}\right)_{+}^{\alpha}-\left(\theta^{\prime} \sum_{t=1}^{k} \Theta_{t}\right)_{+}^{\alpha}\right] .
$$

Proof. We start by observing that each $b_{k}(\theta)$ can be expressed in terms of the spectral tail process $\left(\Theta_{t}\right)$. Indeed, $\left(\mathbf{R V}_{\alpha}\right)$ yields for every $k \geqslant 1$ and $\theta \in \mathbb{S}^{d-1}$ that

$$
\begin{aligned}
b_{k}(\theta) & =\lim _{x \rightarrow \infty} \frac{\mathbb{P}\left(\theta^{\prime} S_{k}>x\right)}{\mathbb{P}(|X|>x)} \\
& =\lim _{x \rightarrow \infty} \frac{\mathbb{P}\left(\cup_{j=1}^{k}\left\{\theta^{\prime} S_{k}>x, \theta^{\prime} X_{j}>x / k\right\} \cap\left\{\theta^{\prime} X_{i}<x / k, 1 \leqslant i<j\right\}\right)}{\mathbb{P}(|X|>x)} \\
& =\lim _{x \rightarrow \infty} \sum_{j=1}^{k}\left[\frac{\mathbb{P}\left(\theta^{\prime} S_{k}>x, \theta^{\prime} X_{j}>x / k\right)}{\mathbb{P}(|X|>x)}-\frac{\mathbb{P}\left(\theta^{\prime} S_{k}>x, \theta^{\prime} X_{j}>x / k, \max _{1 \leqslant i<j} \theta^{\prime} X_{i}>x / k\right)}{\mathbb{P}(|X|>x)}\right] .
\end{aligned}
$$


By stationarity, the summands in the above expression can be written in the form

$$
\begin{aligned}
& \frac{\mathbb{P}\left(\left|X_{0}\right|>x / k\right)}{\mathbb{P}\left(\left|X_{0}\right|>x\right)}\left[\mathbb{P}\left(\theta^{\prime} \sum_{t=1-j}^{k-j} X_{t}>x, \theta^{\prime} X_{0}>x / k|| X_{0} \mid>x / k\right)\right. \\
& \left.-\mathbb{P}\left(\theta^{\prime} \sum_{t=1-j}^{k-j} X_{t}>x, \theta^{\prime} X_{0}>x / k, \max _{1-j \leqslant i<0} \theta^{\prime} X_{i}>x / k|| X_{0} \mid>x / k\right)\right] .
\end{aligned}
$$

Here we used the fact that $\left\{\theta^{\prime} X_{0}>x / k\right\} \subset\left\{\left|X_{0}\right|>x / k\right\}$. Letting $x \rightarrow \infty$ in the above expressions, applying the conditional limits (2.4) and observing that $\mathbb{P}\left(\left|Y_{0}\right|>y\right)=y^{-\alpha}, y>1$, we obtain the limiting expressions

$$
\begin{aligned}
k^{\alpha}[ & \mathbb{P}\left(\left|Y_{0}\right| \theta^{\prime} \sum_{t=1-j}^{k-j} \Theta_{t}>k,\left|Y_{0}\right| \theta^{\prime} \Theta_{0}>1\right) \\
& \left.-\mathbb{P}\left(\left|Y_{0}\right| \theta^{\prime} \sum_{t=1-j}^{k-j} \Theta_{t}>1,\left|Y_{0}\right| \theta^{\prime} \Theta_{0}>1,\left|Y_{0}\right| \max _{1-j \leqslant i<0} \theta^{\prime} \Theta_{i}>1\right)\right] \\
= & \mathbb{E}\left[\left(\theta^{\prime} \sum_{t=1-j}^{k-j} \Theta_{t}\right)_{+}^{\alpha} \wedge\left(k \theta^{\prime} \Theta_{0}\right)_{+}^{\alpha}\right]-\mathbb{E}\left[\left(\theta^{\prime} \sum_{t=1-j}^{k-j} \Theta_{t}\right)_{+}^{\alpha} \wedge\left(k \theta^{\prime} \Theta_{0}\right)_{+}^{\alpha} \wedge \max _{1-j \leqslant i<0}\left(k \theta^{\prime} \Theta_{i}\right)_{+}^{\alpha}\right] .
\end{aligned}
$$

Hence $b_{k}(\theta)$ has representation

$$
b_{k}(\theta)=\sum_{j=1}^{k} \mathbb{E}\left[\left(\left(\theta^{\prime} \sum_{t=1-j}^{k-j} \Theta_{t}\right)_{+}^{\alpha}-\max _{1-j \leqslant i<0}\left(k \theta^{\prime} \Theta_{i}\right)_{+}^{\alpha}\right)_{+} \wedge\left(\left(k \theta^{\prime} \Theta_{0}\right)_{+}^{\alpha}-\max _{1-j \leqslant i<0}\left(k \theta^{\prime} \Theta_{i}\right)_{+}^{\alpha}\right)_{+}\right],
$$

and therefore

$$
\begin{aligned}
b_{k+1}(\theta) & -b_{k}(\theta) \\
=\mathbb{E} & {\left[\left(\theta^{\prime} \sum_{t=0}^{k} \Theta_{t}\right)_{+}^{\alpha} \wedge\left(k \theta^{\prime} \Theta_{0}\right)_{+}^{\alpha}\right] } \\
+ & \sum_{j=1}^{k} \mathbb{E}\left[\left(\left(\theta^{\prime} \sum_{t=-j}^{k-j} \Theta_{t}\right)_{+}^{\alpha}-\max _{-j \leqslant i<0}\left(k \theta^{\prime} \Theta_{i}\right)_{+}^{\alpha}\right)_{+} \wedge\left(\left(k \theta^{\prime} \Theta_{0}\right)_{+}^{\alpha}-\max _{-j \leqslant i<0}\left(k \theta^{\prime} \Theta_{i}\right)_{+}^{\alpha}\right)_{+}\right. \\
& \left.-\left(\left(\theta^{\prime} \sum_{t=1-j}^{k-j} \Theta_{t}\right)_{+}^{\alpha}-\max _{1-j \leqslant i<0}\left(k \theta^{\prime} \Theta_{i}\right)_{+}^{\alpha}\right)_{+} \wedge\left(\left(k \theta^{\prime} \Theta_{0}\right)_{+}^{\alpha}-\max _{1-j \leqslant i<0}\left(k \theta^{\prime} \Theta_{i}\right)_{+}^{\alpha}\right)_{+}\right] .
\end{aligned}
$$

The expectations in the sum are of the type $\mathbb{E} f\left(\Theta_{-s}, \ldots, \Theta_{t}\right)$ for integrable $f$ such that $f\left(x_{-s}, \ldots, x_{t}\right)=0$ if $x_{-s}=0, s, t \geqslant 0$. Then, according to Theorem 3.1 (iii) in [6],

$$
\mathbb{E} f\left(\Theta_{-s}, \ldots, \Theta_{t}\right)=E\left(f\left(\Theta_{0} /\left|\Theta_{s}\right|, \ldots, \Theta_{t+s} /\left|\Theta_{s}\right|\right)\left|\Theta_{s}\right|^{\alpha}\right), \quad s, t \geqslant 0 .
$$

Application of this formula and the fact that our functions $f$ are homogeneous of order $\alpha$ yield

$$
\begin{aligned}
b_{k+1}(\theta)-b_{k}(\theta)= & \mathbb{E}\left[\left(\theta^{\prime} \sum_{t=0}^{k} \Theta_{t}\right)_{+}^{\alpha} \wedge\left(k \theta^{\prime} \Theta_{0}\right)_{+}^{\alpha}\right] \\
& +\sum_{j=1}^{k} \mathbb{E}\left[\left(\left(\theta^{\prime} \sum_{t=0}^{k} \Theta_{t}\right)_{+}^{\alpha}-\max _{0 \leqslant i<j}\left(k \theta^{\prime} \Theta_{i}\right)_{+}^{\alpha}\right)_{+} \wedge\left(\left(k \theta^{\prime} \Theta_{j}\right)_{+}^{\alpha}-\max _{0 \leqslant i<j}\left(k \theta^{\prime} \Theta_{i}\right)_{+}^{\alpha}\right)_{+}\right.
\end{aligned}
$$




$$
\begin{aligned}
&\left.-\left(\left(\theta^{\prime} \sum_{t=1}^{k} \Theta_{t}\right)_{+}^{\alpha}-\max _{1 \leqslant i<j}\left(k \theta^{\prime} \Theta_{i}\right)_{+}^{\alpha}\right)_{+} \wedge\left(\left(k \theta^{\prime} \Theta_{j}\right)_{+}^{\alpha}-\max _{1 \leqslant i<j}\left(k \theta^{\prime} \Theta_{i}\right)_{+}^{\alpha}\right)_{+}\right] \\
&= \mathbb{E}\left[\sum_{j=0}^{k}\left(\left(\theta^{\prime} \sum_{t=0}^{k} \Theta_{t}\right)_{+}^{\alpha}-\max _{0 \leqslant i<j}\left(k \theta^{\prime} \Theta_{i}\right)_{+}^{\alpha}\right)_{+} \wedge\left(\left(k \theta^{\prime} \Theta_{j}\right)_{+}^{\alpha}-\max _{0 \leqslant i<j}\left(k \theta^{\prime} \Theta_{i}\right)_{+}^{\alpha}\right)_{+}\right. \\
&\left.-\sum_{j=1}^{k}\left(\left(\theta^{\prime} \sum_{t=1}^{k} \Theta_{t}\right)_{+}^{\alpha}-\max _{1 \leqslant i<j}\left(k \theta^{\prime} \Theta_{i}\right)_{+}^{\alpha}\right)_{+} \wedge\left(\left(k \theta^{\prime} \Theta_{j}\right)_{+}^{\alpha}-\max _{1 \leqslant i<j}\left(k \theta^{\prime} \Theta_{i}\right)_{+}^{\alpha}\right)_{+}\right] \\
&=\mathbb{E}\left[\left(\theta^{\prime} \sum_{t=0}^{k} \Theta_{t}\right)_{+}^{\alpha}-\left(\theta^{\prime} \sum_{t=1}^{k} \Theta_{t}\right)_{+}^{\alpha}\right] .
\end{aligned}
$$

The last identity follows because there exists $\ell=\min \left\{1 \leqslant j \leqslant n ;\left(k \theta^{\prime} \Theta_{j}\right)_{+}^{\alpha} \geqslant\left(\theta^{\prime} \sum_{t=1}^{k} \Theta_{t}\right)_{+}^{\alpha}\right\}$ such that

$$
\left(\left(\theta^{\prime} \sum_{t=1}^{k} \Theta_{t}\right)_{+}^{\alpha}-\max _{1 \leqslant i<j}\left(k \theta^{\prime} \Theta_{i}\right)_{+}^{\alpha}\right)_{+}=0 \text { for all } j>\ell,
$$

and then also

$$
\begin{aligned}
& \left(\left(\theta^{\prime} \sum_{t=1}^{k} \Theta_{t}\right)_{+}^{\alpha}-\max _{1 \leqslant i<\ell}\left(k \theta^{\prime} \Theta_{i}\right)_{+}^{\alpha}\right)_{+} \wedge\left(\left(k \theta^{\prime} \Theta_{\ell}\right)_{+}^{\alpha}-\max _{1 \leqslant i<\ell}\left(k \theta^{\prime} \Theta_{i}\right)_{+}^{\alpha}\right)_{+} \\
= & \left(\theta^{\prime} \sum_{t=1}^{k} \Theta_{t}\right)_{+}^{\alpha}-\max _{1 \leqslant i<\ell}\left(k \theta^{\prime} \Theta_{i}\right)_{+}^{\alpha},
\end{aligned}
$$

and

$$
\begin{aligned}
& \sum_{j=1}^{\ell-1}\left(\left(\theta^{\prime} \sum_{t=1}^{k} \Theta_{t}\right)_{+}^{\alpha}-\max _{1 \leqslant i<j}\left(k \theta^{\prime} \Theta_{i}\right)_{+}^{\alpha}\right)_{+} \wedge\left(\left(k \theta^{\prime} \Theta_{j}\right)_{+}^{\alpha}-\max _{1 \leqslant i<j}\left(k \theta^{\prime} \Theta_{i}\right)_{+}^{\alpha}\right)_{+} \\
= & \sum_{j=1}^{\ell-1}\left(\left(k \theta^{\prime} \Theta_{j}\right)_{+}^{\alpha}-\max _{1 \leqslant i<j}\left(k \theta^{\prime} \Theta_{i}\right)_{+}^{\alpha}\right)_{+} \\
= & \sum_{j=1}^{\ell-1} \max _{1 \leqslant i \leqslant j}\left(k \theta^{\prime} \Theta_{i}\right)_{+}^{\alpha}-\max _{1 \leqslant i<j}\left(k \theta^{\prime} \Theta_{i}\right)_{+}^{\alpha} \\
= & \max _{1 \leqslant i<\ell}\left(k \theta^{\prime} \Theta_{i}\right)_{+}^{\alpha} .
\end{aligned}
$$

The remainder of this paper crucially depends on the notion of cluster index of the regularly varying sequence $\left(X_{t}\right)$, given as the limiting function:

$$
b(\theta)=\lim _{k \rightarrow \infty}\left(b_{k+1}(\theta)-b_{k}(\theta)\right), \quad \theta \in \mathbb{S}^{d-1} .
$$

In contrast to the quantities $b_{k}(\theta)$ the existence of the limits $b(\theta)$ is not straightforward. The following result yields a sufficient condition for the existence of $b$.

Theorem 3.2. Assume that $\left(X_{t}\right)$ satisfies $\left(\mathbf{R V}_{\alpha}\right)$ for some $\alpha>0$ and that $X_{t}=f\left(\Phi_{t}\right), t \in \mathbb{Z}$, where $f$ is an $\mathbb{R}^{d}$-valued function acting on the Markov chain $\left(\Phi_{t}\right)$ satisfying $\left(\mathbf{D C}_{p}\right)$ for some positive 
$p \in(\alpha-1, \alpha)$. Then the limits

$$
b(\theta)=\mathbb{E}\left[\left(\sum_{t \geqslant 0} \theta^{\prime} \Theta_{t}\right)_{+}^{\alpha}-\left(\sum_{t \geqslant 1} \theta^{\prime} \Theta_{t}\right)_{+}^{\alpha}\right], \quad \theta \in \mathbb{S}^{d-1},
$$

exist and are finite.

Remark 3.3. The cluster index $b$ of $\left(X_{t}\right)$ is a continuous function on $\mathbb{S}^{d-1}$. This is shown in the proof below: $b$ is the uniform limit of continuous functions on $\mathbb{S}^{d-1}$. The index $b(\theta)$ is non-negative since it coincides with the Cèsaro mean $\lim _{k \rightarrow \infty} k^{-1} b_{k}(\theta)$. For $0<\alpha \leqslant 1$, the sub-additivity of the function $x \rightarrow x_{+}^{\alpha}$ implies the inequality $b(\theta) \leqslant \mathbb{E}\left[\left(\theta^{\prime} \Theta_{0}\right)_{+}^{\alpha}\right]$. Moreover, if $\mathbb{E}\left[\left(\theta^{\prime} \Theta_{0}\right)_{+}^{\alpha}\right]>0$ then $b(\theta)>0$ by an application of the mean value theorem when $0<\alpha \leqslant 1$. These two properties are shared by the extremal index of a multivariate stationary process. The extremal index admits a similar representation in terms of the spectral tail process, i.e. $\mathbb{E}\left[\left(\sup _{t \geqslant 0} \theta^{\prime} \Theta_{t}\right)_{+}^{\alpha}-\left(\sup _{t \geqslant 1} \theta^{\prime} \Theta_{t}\right)_{+}^{\alpha}\right]$; see [5].

Remark 3.4. The limit $b$ also exists for various classes of regularly varying stationary processes beyond functions of a Markov chain; see [2, 40, for such examples in the case $d=1$. The cluster index $b$ plays a crucial role for characterizing weak and large deviation limits for partial sums of the processes $\left(X_{t}\right)$. This was recognized in [2, 40, and we extend some of these results to the multivariate case in Section 4.

Proof. We will show that the limit $b(\theta)$ of (3.1) exists as $k \rightarrow \infty$. We start with the case $\alpha>1$. Then, for $x, y \in \mathbb{R}$, by the mean value theorem, $\left|(x+y)_{+}^{\alpha}-x_{+}^{\alpha}\right| \leqslant\left(\alpha|y||x+\xi y|^{\alpha-1}\right) \vee|y|^{\alpha}$ for some $\xi \in(0,1)$. Hence, since $\left|\theta^{\prime} \Theta_{0}\right| \leqslant 1$ a.s.,

$$
\begin{aligned}
\left|b_{k+1}(\theta)-b_{k}(\theta)\right| & \leqslant \mathbb{E}\left[\left(\alpha\left|\theta^{\prime} \Theta_{0}\right|\left|\sum_{t=1}^{k} \theta^{\prime} \Theta_{t}+\xi \theta^{\prime} \Theta_{0}\right|^{\alpha-1}\right) \vee\left|\theta^{\prime} \Theta_{0}\right|^{\alpha}\right] \\
& \leqslant \mathbb{E}\left[\left(\alpha\left|\sum_{t=1}^{k} \theta^{\prime} \Theta_{t}+\xi \theta^{\prime} \Theta_{0}\right|^{\alpha-1}\right) \vee 1\right]=I_{0} .
\end{aligned}
$$

For $\alpha \in(1,2]$,

$$
I_{0} \leqslant 1+\alpha \sum_{t=0}^{k} \mathbb{E}\left|\theta^{\prime} \Theta_{t}\right|^{\alpha-1} .
$$

We will show that the right-hand side is finite, implying that $\mathbb{E}\left|\sum_{t=0}^{\infty}\right| \theta^{\prime} \Theta_{t}||^{\alpha-1}<\infty$ and $\sum_{t=0}^{\infty} \theta^{\prime} \Theta_{t}$ converges absolutely a.s. An application of Lebesgue dominated convergence shows that the limit $b(\theta)$ exists and is finite. For $\alpha>2$, an application of Minkowski's inequality yields

$$
I_{0} \leqslant 1+\alpha\left(\sum_{t=0}^{k}\left(\mathbb{E}\left|\theta^{\prime} \Theta_{t}\right|^{\alpha-1}\right)^{1 /(\alpha-1)}\right)^{\alpha-1} .
$$

We will show that the right-hand side is finite and then the same argument as for $\alpha \in(1,2]$ applies. We will achieve the bounds for $I_{0}$ by showing that there exists $c>0$ such that

$$
\mathbb{E}\left|\theta^{\prime} \Theta_{t}\right|^{\alpha-1} \leqslant c \beta^{t}, \quad t \geqslant 0 .
$$

Using the fact that $\Theta_{t}=Y_{t} / Y_{0}$ and $Y_{0}$ are independent, for $t \geqslant 1$ and $s=\alpha-1$,

$$
\mathbb{E}\left|Y_{0}\right|^{s} \mathbb{E}\left|\theta^{\prime} \Theta_{t}\right|^{s} \leqslant \mathbb{E}\left|Y_{0}\right|^{s} \mathbb{E}\left|\Theta_{t}\right|^{s}=\mathbb{E}\left|Y_{t}\right|^{s} .
$$


By definition of the tail process and Markov's inequality, for small $\epsilon>0$ such that $s(1+\epsilon)<\alpha$,

$$
\begin{aligned}
\mathbb{E}\left|Y_{t}\right|^{s} & =\int_{0}^{\infty} \mathbb{P}\left(\left|Y_{t}\right|^{s}>y\right) d y \\
& =\int_{0}^{\infty} \lim _{x \rightarrow \infty} \mathbb{P}\left(\left|x^{-1} X_{t}\right|^{s}>y|| X_{0} \mid>x\right) d y \\
\leqslant & \int_{1}^{\infty} y^{-(1+\epsilon)} d y \lim _{x \rightarrow \infty} \frac{\mathbb{E}\left[\left|X_{t}\right|^{s(1+\epsilon)} \mathbb{1}_{\left\{\left|X_{0}\right|>x\right\}}\right]}{x^{s(1+\epsilon)} \mathbb{P}\left(\left|X_{0}\right|>x\right)} \\
& +\int_{0}^{1} y^{-(1-\epsilon)} d y \lim _{x \rightarrow \infty} \frac{\mathbb{E}\left[\left|X_{t}\right|^{s(1-\epsilon)} \mathbb{1}_{\left\{\left|X_{0}\right|>x\right\}}\right]}{x^{s(1-\epsilon)} \mathbb{P}\left(\left|X_{0}\right|>x\right)} \\
= & R_{1}+R_{2} .
\end{aligned}
$$

By virtue of $\left(\mathbf{D C}_{p}\right)$ for some $p \in(\alpha-1, \alpha)$, using a recursive argument, we obtain for sufficiently large $y$ and $s(1+\epsilon) \leqslant p$,

$$
\mathbb{E}\left[\left|X_{t}\right|^{s(1+\epsilon)} \mid \Phi_{0}=y\right] \leqslant \beta^{t}|f(y)|^{s(1+\epsilon)}+b \sum_{j=1}^{t} \beta^{j} .
$$

Using this inequality and Karamata's theorem (see [7), for some $c>0$,

$$
\begin{aligned}
R_{1} & \leqslant c \lim _{x \rightarrow \infty} \frac{\mathbb{E}\left[\mathbb{1}_{\left\{\left|X_{0}\right|>x\right\}} \mathbb{E}\left[\left|X_{t}\right|^{s(1+\epsilon)} \mid \Phi_{0}\right]\right]}{x^{s(1+\epsilon)} \mathbb{P}\left(\left|X_{0}\right|>x\right)} \\
& \leqslant c \beta^{t} \lim _{x \rightarrow \infty} \frac{\mathbb{E}\left[\left|X_{0}\right|^{s(1+\epsilon)} \mathbb{1}_{\left\{\left|X_{0}\right|>x\right\}}\right]}{x^{s(1+\epsilon)} \mathbb{P}\left(\left|X_{0}\right|>x\right)} \leqslant c \beta^{t} .
\end{aligned}
$$

Similarly, $R_{2} \leqslant c \beta^{t}$. We conclude that (3.1) holds for $\alpha>1$.

It remains to consider the case $\alpha \leqslant 1$. We observe that $\left|(x+y)_{+}^{\alpha}-x_{+}^{\alpha}\right| \leqslant|y|^{\alpha}$ for any $x, y \in \mathbb{R}$. Hence

$$
\left|b_{k+1}(\theta)-b_{k}(\theta)\right| \leqslant \mathbb{E}\left|\theta^{\prime} \Theta_{0}\right|^{\alpha} \leqslant 1 .
$$

It suffices to show that $\sum_{t=0}^{\infty}\left|\theta^{\prime} \Theta_{t}\right|<\infty$ a.s. This follows if $\sum_{t=0}^{\infty} \mathbb{E}\left|\theta^{\prime} \Theta_{t}\right|^{s}<\infty$ for some $s<p$. The proof is analogous, using $\left(\mathbf{D C}_{p}\right)$ for some $p<\alpha$.

\section{Limit theory For FUnCtions of REgenerative Markov Chains}

In this section we present the main results of this paper. Throughout we consider an $\mathbb{R}^{d}$-valued process $X_{t}=f\left(\Phi_{t}\right), t \in \mathbb{Z}$, where $\left(\Phi_{t}\right)$ is an irreducible aperiodic Markov chain. We present two types of limit results for the partial sums $\left(S_{n}\right)$ of $\left(X_{n}\right)$ : central limit theory with infinite stable limits in Theorem 4.1 and precise large deviation results in Theorem 4.3. The proofs of these results are postponed to Sections 6 and 7

4.1. Stable limit theory. We start with a central limit theorem with stable limit law.

Theorem 4.1. Consider an $\mathbb{R}^{d}$-valued strictly stationary sequence $\left(X_{t}\right)=\left(f\left(\Phi_{t}\right)\right)$ satisfying the following conditions:

- $\left(\mathbf{R V}_{\alpha}\right)$ for some $\alpha \in(0,2), \mathbb{E} X=0$ if $\alpha>1$ and $X$ is symmetric if $\alpha=1$.

- $\left(\mathbf{D C}_{p}\right)$ for some $p \in((\alpha-1) \vee 0, \alpha)$.

Let $\left(a_{n}\right)$ be a sequence of positive numbers such that $n \mathbb{P}\left(\left|X_{0}\right|>a_{n}\right) \sim 1$. Then the following statements hold: 
(1) The central limit theorem $a_{n}^{-1} S_{n} \stackrel{d}{\rightarrow} \xi_{\alpha}$ is satisfied for a centered $\alpha$-stable random vector $\xi_{\alpha}$ with spectral measure $\Gamma_{\alpha}$ on $\mathbb{S}^{d-1}$ (see [50], Section 2.3, for a definition) given by the relation

$$
b(\theta)=C_{\alpha} \int_{\mathbb{S}^{d-1}}\left(\theta^{\prime} s\right)_{+}^{\alpha} \Gamma_{\alpha}(d s), \quad \theta \in \mathbb{S}^{d-1},
$$

where $b$ is the cluster index of $\left(X_{t}\right)$ introduced in Section 3 and

$$
C_{\alpha}=\frac{1-\alpha}{\Gamma(2-\alpha) \cos (\pi \alpha / 2)}
$$

If $b \equiv 0$ the limit $\xi_{\alpha}=0$ a.s.

(2) If $b \neq 0$ the partial sums over full cycles $(S(i))_{i=1,2, \ldots}$ defined in (2.5) are regularly varying with index $\alpha$ and spectral measure $\mathbb{P}_{\Theta^{\prime}}(\cdot)$ on $\mathbb{S}^{d-1}$ given by

$$
d \mathbb{P}_{\Theta^{\prime}}(d s)=\frac{b(s)}{\int_{\mathbb{S}^{d-1}} b(\theta) d \mathbb{P}_{\Theta}(\theta)} d \mathbb{P}_{\Theta}(d s) .
$$

The proof of Theorem 4.1 is given in Section 6. To a large extent, the results of Theorem 4.1 can be extended to the case of non-irreducible Markov chains. A short discussion of this topic will be given at the end of Section 6 .

A discussion of related stable limit results. Theorem 4.1 complements the central limit theorem with Gaussian limits for $\mathbb{R}^{d}$-valued functions of a Markov chain; see Theorem 2.2 above. For both results, conditions of type $\left(\mathbf{D C}_{p}\right)$ enter the proofs to show the existence of moments of $S(1)$ under the existence of the corresponding moments for $X_{0}$.

The history of stable limit theory for non-linear multivariate time series is short in comparison with the finite variance case. Davis and Mikosch 17] prove a central limit theorem with $\alpha$-stable limit for an $\mathbb{R}^{d}$-valued strictly stationary sequence $\left(X_{t}\right)$, satisfying a weak dependence condition. The result is a straightforward extension of the 1-dimensional result proved in Theorem 3.1 of Davis and Hsing [16. We recall the forementioned results for the reason of comparison with Theorem 4.1

Theorem 4.2. Assume that the strictly stationary $\mathbb{R}^{d}$-valued sequence $\left(X_{t}\right)$ satisfies $\left(\mathbf{R V}_{\alpha}\right)$ for some $\alpha>0$ and the following point process convergence result holds:

$$
N_{n}=\sum_{t=1}^{n} \delta_{a_{n}^{-1} X_{t}} \stackrel{d}{\rightarrow} N=\sum_{i=1}^{\infty} \sum_{j=1}^{\infty} \delta_{P_{i} Q_{i j}},
$$

where $\left(P_{i}\right)$ are the points of a Poisson random measure on $(0, \infty)$ with intensity $h(y)=\gamma \alpha y^{-\alpha-1}$, $y>0$, and it is assumed that $\gamma>011$ the sequence $\left(Q_{i j}\right)_{j \geqslant 1}, i=1,2, \ldots$, is iid with values $\left|Q_{i j}\right| \leqslant 1$, independent of $\left(P_{i}\right)$ and such that $\sup _{j \geqslant 1}\left|Q_{i j}\right|=1$.

(1) If $\alpha \in(0,1)$ then

$$
a_{n}^{-1} S_{n} \stackrel{d}{\rightarrow} \xi_{\alpha}=\sum_{i=1}^{\infty} \sum_{j=1}^{\infty} P_{i} Q_{i j}
$$

and $\xi_{\alpha}$ has an $\alpha$-stable distribution,

\footnotetext{
${ }^{1}$ Basrak and Segers [6], Proposition 4.2, show that $\gamma>0$ is automatic if $\left(X_{t}\right)$ satisfies their anti-clustering Condition 4.1 and a modification of the mixing $\mathcal{A}\left(a_{n}\right)$ from [16]. Both conditions are very mild. The quantity $\gamma$ is known as the extremal index of the sequence $\left(X_{t}\right)$; see 35 .
} 
(2) If $\alpha \in[1,2)$ and for any $\delta>0$,

$$
\lim _{\varepsilon \downarrow 0} \limsup _{n \rightarrow \infty} \mathbb{P}\left(\left|S_{n}(0, \varepsilon]-\mathbb{E} S_{n}(0, \varepsilon]\right|>\delta\right)=0,
$$

where $S_{n}(0, \varepsilon]=a_{n}^{-1} \sum_{t=1}^{n} X_{t} \mathbb{1}_{\left\{\left|X_{t}\right| \leqslant \varepsilon a_{n}\right\}}$, then

$$
a_{n}^{-1} S_{n}-\mathbb{E} S_{n}(0,1] \stackrel{d}{\rightarrow} \xi_{\alpha},
$$

where $\xi_{\alpha}$ is the distributional limit as $\varepsilon \downarrow 0$ of

$$
\left(\sum_{i=1}^{\infty} \sum_{j=1}^{\infty} P_{i} Q_{i j} \mathbb{1}_{(\varepsilon, \infty)}\left(P_{i}\left|Q_{i j}\right|\right)-\int_{\varepsilon<|x| \leqslant 1} x \mu_{X}(d x)\right)
$$

which exists and has an $\alpha$-stable distribution. (Recall that $\mu_{X}$ is the limit measure in (2.2).)

The latter result has been the basis for a variety of results for partial sums of strictly stationary processes with infinite variance stable limits; see [17, 37, 5, 52. The main idea of the proof of Theorem 4.2 is a continuous mapping argument acting on $N_{n} \stackrel{d}{\rightarrow} N$, showing that the sums of the points of $N_{n}$ converge in distribution to the corresponding sum of the points of $N$. This method is rather elegant and can be applied to a large variety of strictly stationary regularly varying vector sequences $\left(X_{t}\right)$. The proofs use advanced point process techniques.

A characterization of the parameters of the distribution of the multivariate limit $\xi_{\alpha}$ in Theorem 4.2 can be given by extending Theorem 3.2 in [16] to the multidimensional case: if

$$
\mathbb{E}\left(\sum_{j \geqslant 1}\left|Q_{1 j}\right|\right)^{\alpha}<\infty
$$

then the Lévy spectral measure $\Gamma_{\alpha}$ of $\xi_{\alpha}$ is described by

$$
\int_{\mathbb{S}^{d-1}}\left(\theta^{\prime} s\right)_{+}^{\alpha} \Gamma_{\alpha}(d s)=\gamma \frac{\alpha}{2-\alpha} \mathbb{E}\left[\left(\sum_{t \geqslant 1} \theta^{\prime} Q_{1 t}\right)_{+}^{\alpha}\right], \quad \theta \in \mathbb{S}^{d-1} .
$$

This representation is particularly useful for $\alpha<1$. Then (4.5) is always satisfied. Adapting Theorem 4.2 in terms of the tail process as in Basrak et al. [5], an alternative characterization of the Lévy spectral measure $\Gamma_{\alpha}$ is the following: if

$$
\mathbb{E}\left(\sum_{t \geqslant 0}\left|\Theta_{t}\right|\right)^{\alpha}<\infty
$$

then

$$
\int_{\mathbb{S}^{d-1}}\left(\theta^{\prime} s\right)_{+}^{\alpha} \Gamma_{\alpha}(d s)=C_{\alpha}^{-1} \mathbb{E}\left[\left(\sum_{t \geqslant 0} \theta^{\prime} \Theta_{t}\right)_{+}^{\alpha} \mathbb{1}_{\left\{\Theta_{i}=0, \forall i \leqslant-1\right\}}\right], \quad \theta \in \mathbb{S}^{d-1} .
$$

Conditions (4.5) and (4.6) may fail for $\alpha>1$, e.g. for a $\operatorname{GARCH}(1,1)$ model; see Section 5.4.

If we assume the conditions of Theorem 4.1, classical computation for $\alpha \neq 1$ yields

$$
\begin{aligned}
\mathbb{E}\left[\exp \left(i v^{\prime} \xi_{\alpha}\right)\right] & =\exp \left(-\int_{\mathbb{S}^{d-1}}\left|v^{\prime} \theta\right|^{\alpha}\left(1-i \operatorname{sign}\left(v^{\prime} \theta\right) \tan (\pi \alpha / 2)\right) \Gamma_{\alpha}(d \theta)\right) \\
& =\exp \left(\int_{0}^{\infty} \mathbb{E}\left[\exp \left(i u \sum_{t=1}^{\infty} v^{\prime} \Theta_{t}\right)-\exp \left(i u \sum_{t=0}^{\infty} v^{\prime} \Theta_{t}\right)\right] \alpha x^{-\alpha-1} d x\right) .
\end{aligned}
$$

For $\alpha \in(0,1)$, this form of the limiting stable characteristic function was proved in Basrak and Segers [6].

The additional condition (4.4) is not easily checked for dependent sequences. It is implied for stationary $\rho$-mixing processes with rate function $\rho(j)$ satisfying $\sum_{j \geqslant 1} \rho\left(2^{j}\right)<\infty$; see [29]. It is 
also implied by $\left(\mathbf{D C}_{p}\right)$ for functions of an irreducible Markov chain; see [40. For a (possibly nonirreducible) Markov chain $\left(X_{t}\right)$, condition $\left(\mathbf{D C}_{p}\right)$ is much weaker than this $\rho$-mixing condition which is equivalent to a spectral gap in $L^{2}(\mathbb{P})$; see [36].

In our paper, characteristic function based methods are employed which are close to those used in classical limit theory for iid sequences; see e.g. 44. As in the iid case, Theorem 4.1 yields an explicit form of the characteristic function of the limiting $\alpha$-stable random vector. The underlying extremal dependence structure of $\left(X_{t}\right)$ shows via the cluster index $b(\theta)$ which appears explicitly in the characteristic function. We refer the reader to the extensive discussion in [2] on the comparison of the point process and the characteristic function approaches to stable limit theory. One drawback of our approach is that, in contrast to the point process approach, we do not have series representations of $\xi_{\alpha}$ in terms of the sequence $\left(\Theta_{t}\right)$.

Recently, the special case of solutions to multivariate stochastic recurrence equations (5.3) has attracted attention; see e.g. [15, 12. In this case, one can exploit the underlying random iterative contractive structure to derive stable limits without additional restrictions. We mention that drift conditions such as $\left(\mathbf{D C}_{p}\right)$ are automatically satisfied for solutions of stochastic recurrence equations; see Section 5 .

4.2. Precise large deviations for functions of a Markov chain. In this section, we extend some of the results obtained in 40 for general univariate regularly varying sequences 3 We again focus on $\mathbb{R}^{d}$-valued sequences $\left(X_{t}\right)=\left(f\left(\Phi_{t}\right)\right)$ for an underlying aperiodic irreducible Markov chain $\left(\Phi_{t}\right)$. The case $\alpha \in(0,2)$ turns out to be a consequence of Theorem 4.1] the proof is given in Section 7.1. The proof in the case $\alpha>2$ is more involved and requires different techniques; see Section 7.2 .

Theorem 4.3. Consider an $\mathbb{R}^{d}$-valued strictly stationary sequence $\left(X_{t}\right)=\left(f\left(\Phi_{t}\right)\right)$ for an aperiodic irreducible Markov chain $\left(\Phi_{t}\right)$. Assume that $\left(X_{t}\right)$ satisfies the condition $\left(\mathbf{R V}_{\alpha}\right)$ for some $\alpha>0$. Let $\left(\lambda_{n}\right)$ be any sequence such that $\log \left(\lambda_{n}\right)=o(n)$ and $\lambda_{n} / n^{1 / \alpha+\varepsilon} \rightarrow \infty$ if $\alpha \in(0,2)$ and $\lambda_{n} / n^{0.5+\varepsilon} \rightarrow \infty$ if $\alpha>2$ for any $\varepsilon>0$. Assume either

(1) $\alpha \in(0,2)$ and the conditions of Theorem 4.1 are satisfied, or

(2) $\alpha>2, \alpha \notin \mathbb{N}$ or $b(\theta)=b(-\theta), \theta \in \mathbb{S}^{d-1}$, and $\left(\mathbf{D C}_{p}\right)$ holds for every $p<\alpha$,

then the following large deviation principle holds:

$$
\frac{\mathbb{P}\left(\lambda_{n}^{-1} S_{n} \in \cdot\right)}{n \mathbb{P}\left(|X|>\lambda_{n}\right)} \stackrel{v}{\rightarrow} \nu_{\alpha}, \quad n \rightarrow \infty
$$

where $\nu_{\alpha}$ is a Radon measure on the Borel $\sigma$-field of $\overline{\mathbb{R}}_{0}^{d}$ uniquely determined by the relations

$$
\nu_{\alpha}\left(t\left\{x: \theta^{\prime} x>1\right\}\right)=t^{-\alpha} \nu_{\alpha}\left(\left\{x: \theta^{\prime} x>1\right\}\right)=t^{-\alpha} b(\theta), \quad \theta \in \mathbb{S}^{d-1}, t>0 .
$$

Remark 4.4. The conditions $\alpha \notin \mathbb{N}$ or $b(\cdot)=b(-\cdot)$ are needed to apply inverse results for regular variation. For $\alpha>2$, we show that the measure $\nu_{\alpha}$ on the Borel $\sigma$-field of $\overline{\mathbb{R}}_{0}^{d}$ is uniquely determined by its values on sets of the form $t\left\{x: \theta^{\prime} x>1\right\}, t>0, \theta \in \mathbb{S}^{d-1}$, provided the mentioned additional conditions are met. In general, such conditions cannot be avoided; 32, 26] give counterexamples for integer values $\alpha$. In [3, 8, 33 further conditions on the vector $X$ are given which allow one to discover the measure $\nu_{\alpha}$ from its knowledge on the sets $t\left\{x: \theta^{\prime} x>1\right\}, t>0, \theta \in \mathbb{S}^{d-1}$.

Remark 4.5. The proof of Theorem 4.3 shows that (4.1) holds uniformly for certain intervals of normalizations and for half-spaces not containing the origin. To be precise, the following uniform

${ }^{2}$ For comparison and since we will use it in the proofs, we quote the main result of [40] as Theorem 7.2 
relations hold

$$
\lim _{n \rightarrow \infty} \sup _{x \in \Lambda_{n}}\left|\frac{\mathbb{P}\left(\theta^{\prime} S_{n}>x\right)}{n \mathbb{P}(|X|>x)}-b(\theta)\right|=0, \quad \theta \in \mathbb{S}^{d-1},
$$

for regions $\Lambda_{n}=\left(b_{n}, c_{n}\right)$. Here $\left(b_{n}\right)$ satisfies $b_{n}=n^{0.5+\varepsilon}$ in the case $\alpha>2$ and $b_{n}=n^{1 / \alpha+\varepsilon}$ in the case $\alpha \in(0,2)$ for any $\varepsilon>0$, and $\left(c_{n}\right)$ is chosen such that $c_{n}>b_{n}$ and $\log c_{n}=o(n)$. Moreover, for (4.3) one does not need the additional conditions $b(\cdot)=b(-\cdot)$ and $\alpha \notin \mathbb{N}$.

\section{EXAMPLES}

Here we consider several examples of regularly varying stationary processes with index $\alpha>0$, where the theory of the previous sections applies. In particular, we will determine the tail process $\left(\Theta_{t}\right)$, the cluster index $b$ and verify the drift condition $\left(\mathbf{D C}_{p}\right)$ for $p<\alpha$. All models considered fall in the class of functions acting on an aperiodic irreducible Markov chain.

5.1. Vector-autoregressive process. Consider the vector-autoregressive process of order 1 given by

$$
X_{t}=A X_{t-1}+Z_{t}, \quad t \in \mathbb{Z},
$$

where $A$ is a random $d \times d$ matrix whose eigenvalues are less than 1 in absolute value, and $A$ is independent of the iid $\mathbb{R}^{d}$-valued sequence $\left(Z_{t}\right)$ which is regularly varying with index $\alpha>0$. Then we also have $\mathbb{E}\|A\|^{s}<1$ for every $s>0$. Here $\|\cdot\|$ denotes the operator norm with respect to the Euclidean norm.

Then a stationary solution $\left(X_{t}\right)$ to (5.1) exists and has representation

$$
X_{t}=A^{t} X_{0}+\sum_{i=1}^{t} A^{t-i} Z_{i}, \quad t \geqslant 0
$$

see 11, Chapter 11. Morever, $X_{0}$ is regularly varying with index $\alpha$; see 48. In particular, denoting the limiting measure of the regularly varying vector $Z_{0}$ by $\mu_{Z}$, it follows from [48] that

$$
\frac{\mathbb{P}\left(x^{-1} X_{0} \in \cdot\right)}{\mathbb{P}\left(\left|Z_{0}\right|>x\right)} \stackrel{v}{\rightarrow} \sum_{i=0}^{\infty} \mathbb{E}\left[\mu_{Z}\left(\left\{x \in \mathbb{R}^{d}: A^{i} x \in \cdot\right\}\right)\right] .
$$

Since

$$
\left(X_{1}, \ldots, X_{h}\right)=\left(A, \ldots, A^{h}\right) X_{0}+\left(Z_{1}, \ldots, \sum_{t=1}^{h} A^{h-t} Z_{t}\right),
$$

and $\left(Z_{t}\right)_{t \geqslant 1}$ is independent of $X_{0}$, regular variation of $\left(X_{1}, \ldots, X_{h}\right)$ is a consequence of the fact that regular variation is kept under linear transformations. Let $C$ be a continuity set relative to the limiting measure $\mu_{h+1}$ of $\left(X_{0}, \ldots, X_{h}\right)$ and $I_{d}$ the identity matrix. Since $X_{0}$ is independent of $\left(Z_{t}\right)_{t \geqslant 1}$,

$$
\begin{aligned}
\mathbb{P}\left(x^{-1}\left(X_{0}, \ldots, X_{h}\right) \in C|| X_{0} \mid>x\right)= & \mathbb{P}\left(x^{-1}\left(I_{d}, A, \ldots, A^{h}\right) X_{0} \in C|| X_{0} \mid>x\right) \\
& +\mathbb{P}\left(x^{-1}\left(0, Z_{1}, \sum_{i=1}^{2} A^{2-i} Z_{i}, \ldots, \sum_{i=1}^{h} A^{h-i} Z_{i} \in C\right)+o(1)\right. \\
\rightarrow & \mathbb{P}\left(\left(I_{d}, A, \ldots, A^{h}\right) Y_{0} \in C\right), \quad x \rightarrow \infty .
\end{aligned}
$$

Thus we may identify $\left(\Theta_{t}\right)_{t=0, \ldots, h}$ with $\left(I_{d}, A, \ldots, A^{h}\right) \Theta_{0}$. In view of (5.2),

$\mathbb{P}\left(x^{-1} X_{0} /\left|X_{0}\right| \in \cdot|| X_{0} \mid>x\right) \stackrel{w}{\rightarrow} \frac{\sum_{i=0}^{\infty} \mathbb{E}\left[\mu_{Z}\left(\left\{x \in \mathbb{R}^{d}: A^{i} x /\left|A^{i} x\right| \in \cdot,\left|A^{i} x\right|>1\right\}\right)\right]}{\sum_{i=0}^{\infty} \mathbb{E}\left[\mu_{Z}\left(\left\{x \in \mathbb{R}^{d}:\left|A^{i} x\right|>1\right\}\right)\right]}=\mathbb{P}\left(\Theta_{0} \in \cdot\right)$. 
Writing $\left(I_{d}-A\right)^{-1}=\sum_{t=0}^{\infty} A^{t}$ (this series converges since the largest eigenvalue of $A$ is smaller than 1), we conclude that

$$
b(\theta)=\mathbb{E}\left[\left(\theta^{\prime}\left(I_{d}-A\right)^{-1} \Theta_{0}\right)_{+}^{\alpha}-\left(\theta^{\prime} A\left(I_{d}-A\right)^{-1} \Theta_{0}\right)_{+}^{\alpha}\right], \quad \theta \in \mathbb{S}^{d-1} .
$$

Next we show $\left(\mathbf{D C}_{p}\right)$ for $p<\alpha$. First assume $p>1$. A Taylor series expansion yields

$$
\begin{aligned}
\mathbb{E}\left(\left|A x+Z_{1}\right|^{p}-|A x|^{p}\right) & \leqslant p \mathbb{E}\left[\left|Z_{1}\right|\left|A x+\xi Z_{1}\right|^{p-1}\right] \\
& \leqslant c\left(\mathbb{E}|A x|^{p-1}+1\right) \leqslant c\left(|x|^{p-1}+1\right)
\end{aligned}
$$

for some random variable $\xi \in(0,1)$ a.s. Then for some $\beta \in\left(\mathbb{E}\|A\|^{p}, 1\right)$ and sufficiently large $|x|$,

$$
\mathbb{E}\left|A x+Z_{1}\right|^{p} \leqslant \mathbb{E}|A x|^{p}+c\left(1+|x|^{p-1}\right) \leqslant \mathbb{E}\|A\|^{p}|x|^{p}\left(1+c|x|^{-1}\right)+c \leqslant \beta|x|^{p}+c,
$$

and $\left(\mathbf{D C}_{p}\right)$ is satisfied. If $p \leqslant 1$ a simpler argument applies with $\beta=\mathbb{E}\|A\|$ :

$$
\mathbb{E}\left(\left|A x+Z_{1}\right|^{p} \leqslant \mathbb{E}|A x|^{p}+\mathbb{E}\left|Z_{1}\right|^{p} \leqslant \beta|x|^{p}+c .\right.
$$

If the Markov chain $\left(X_{t}\right)$ is also aperiodic and irreducible the results in Section 4 are directly applicable with $f(x)=x$.

5.2. Random affine mapping. Following Kesten [32, we consider the stochastic recurrence equation

$$
X_{t}=A_{t} X_{t-1}+B_{t}, \quad t \in \mathbb{Z},
$$

where $\left(\left(A_{t}, B_{t}\right)\right)_{t \in \mathbb{Z}}$ is an iid sequence, $A_{t}$ are random $d \times d$-matrices and $B_{t}$ are $\mathbb{R}^{d}$-valued random vectors. We also assume $\mathbb{E} \log ^{+}\|A\|<\infty$, where $\|\cdot\|$ denotes the operator norm with respect to the Euclidean norm, $\mathbb{E} \log ^{+}|B|<\infty$, and that the Lyapunov exponent of the stochastic recurrence equation (5.3) is negative. These conditions ensure that an a.s. unique stationary causal solution $\left(X_{t}\right)$ to (5.3) exists; see 9]. Under additional regularity conditions which ensure that the distribution of $A$ is sufficiently spread out, the equation

$$
\varrho(\kappa)=\lim _{n \rightarrow \infty} n^{-1} \log \mathbb{E}\left\|A_{1} \cdots A_{n}\right\|^{\kappa}=0, \quad \kappa>0,
$$

has a unique positive solution $\alpha$ and $\theta^{\prime} X, \theta \in \mathbb{S}^{d-1}$, is regularly varying with index $\alpha$. Under stronger conditions on $A, \alpha$ can be calculated as the solution to $E\|A\|^{\kappa}=1, \kappa>0$; see [20, 13] for recent results. Kesten 32 had already given conditions which ensured that at least one of the linear combinations $\theta^{\prime} X, \theta \in \mathbb{S}^{d-1}$, is regularly varying with index $\alpha$. In general, one cannot conclude from regular variation of $\theta^{\prime} X, \theta \in \mathbb{S}^{d-1}$, that $X$ is regularly varying; see [32, 26] for some counterexamples 3 In $[3,8,33$, conditions are given which ensure that the regular variation of a vector can be recovered from the regular variation of its linear projections. One of these conditions is that $\alpha \notin \mathbb{N}$; see $\left[3\right.$ for details. In what follows, we will assume that $X_{t}$ is regularly varying with index $\alpha>0$ and that the stronger moment conditions $\mathbb{E}\|A\|^{2(\alpha+\epsilon)}<\infty$ and $\mathbb{E}|B|^{2(\alpha+\epsilon)}<\infty$ hold for some $\epsilon>0$. If $A_{t}$ and $B_{t}$ are independent the milder moment conditions $\mathbb{E}\|A\|^{\alpha+\epsilon}<\infty$ and $\mathbb{E}|B|^{\alpha+\epsilon}<\infty$ for some $\epsilon>0$ suffice.

Calculation yields

$$
X_{t}=\Pi_{t} X_{0}+R_{t}, \quad \text { where } \Pi_{t}=A_{t} \cdots A_{1}, \quad t \geqslant 1,
$$

where $\mathbb{E}\left|R_{t}\right|^{\alpha+\epsilon}<\infty$ and hence

$$
\mathbb{P}\left(x^{-1}\left(X_{0}, \ldots, X_{t}\right) \in \cdot|| X_{0} \mid>x\right) \stackrel{w}{\rightarrow} \mathbb{P}\left(\left|Y_{0}\right|\left(I_{d}, \Pi_{1}, \ldots \Pi_{t}\right) \Theta_{0} \in \cdot\right) .
$$

where $\mathbb{P}\left(X_{0} /\left|X_{0}\right| \in \cdot|| X_{0} \mid>x\right) \stackrel{w}{\rightarrow} \mathbb{P}\left(\Theta_{0} \in \cdot\right)$ and $\Theta_{0}$ is independent of $\left(A_{t}\right)_{t \geqslant 1}$. Therefore $\left(\Theta_{i}\right)_{i=0, \ldots, t}=\left(I_{d}, \Pi_{1}, \ldots, \Pi_{t}\right) \Theta_{0}$. Writing $\Pi_{0}=I_{d}$, the identity matrix in $\mathbb{R}^{d}$, and $\left(Z_{t}\right)$ for the

\footnotetext{
${ }^{3}$ However, it might be possible to prove regular variation of $X_{t}$ by using the structure of 5.3 .
} 
solution of the stochastic recurrence equation (5.3) in the special case $B=I_{d}$, we obtain from Theorem 3.2 ,

$$
\begin{aligned}
b(\theta) & =\mathbb{E}\left[\left(\theta^{\prime} \sum_{t \geqslant 0} \Pi_{t} \Theta_{0}\right)_{+}^{\alpha}-\left(\theta^{\prime} \sum_{t \geqslant 1} \Pi_{t} \Theta_{0}\right)_{+}^{\alpha}\right] \\
& =\mathbb{E}\left[\left(\theta^{\prime}\left(Z_{1}+I_{d}\right) \Theta_{0}\right)_{+}^{\alpha}-\left(\theta^{\prime} Z_{1} \Theta_{0}\right)_{+}^{\alpha}\right], \quad \theta \in \mathbb{S}^{d-1},
\end{aligned}
$$

provided we can show $\left(\mathbf{D C}_{p}\right)$ for the Markov chain $\left(\Phi_{t}\right)=\left(X_{t}\right)$. The formula (5.6) is in agreement with the calculations for $d=1$ in [2].

Since (5.4) is satisfied we can use Lemma 2.1 for proving $\left(\mathbf{D C}_{p}\right)$. Assuming irreducibility and aperiodicity of the Markov chain $\left(X_{t}\right)$ and exploiting the definition of $\alpha$ as solution to (5.4), one can choose $m \geqslant 1$ sufficiently large such that $\mathbb{E}\left\|A_{1} \cdots A_{m}\right\|^{p}<1$ for any $p<\alpha$. Indeed, assume on the contrary that $\varrho(p) \geqslant 0$ for some $p<\alpha$. This contradicts the convexity of $\varrho$ which has roots at 0 and $\alpha$. Then the $m$-skeleton of the chain satisfies the drift condition, $\left(\mathbf{D C}_{p, m}\right)$ follows and Lemma 2.1 yields $\left(\mathbf{D C}_{p}\right)$. Thus we conclude that the results of Section 4 are directly applicable to the Markov chain $\left(X_{t}\right)$ with $f(x)=x$ if it is also aperiodic and irreducible.

5.3. Sample autocovariance function of one-dimensional random affine mapping. Consider the solution $\left(X_{t}\right)$ to the stochastic recurrence equation (5.3) in the case $d=1$, under irreducibility and aperiodicity. We assume the conditions and use the notation of Section 5.2. In addition, we write

$$
\Pi_{s, t}= \begin{cases}A_{s} \cdots A_{t} & s \leqslant t \\ 1 & \text { otherwise. }\end{cases}
$$

In particular, we assume that $\left(X_{t}\right)$ is regularly varying with index $\alpha>0$ satisfying $\mathbb{E}|A|^{\alpha}=1$, $\mathbb{E}|A|^{\alpha+\varepsilon}<\infty$ and $\mathbb{E}|B|^{\alpha+\varepsilon}<\infty$ for some $\varepsilon>0$. For $h \geqslant 0$, consider the process $\Phi_{t}=$ $\left(X_{t}, X_{t-1}, \ldots, X_{t-h}\right)^{\prime}, t \in \mathbb{Z}$, of lagged vectors. They constitute an $\mathbb{R}^{h+1}$-valued stationary, aperiodic and irreducible Markov chain. Similar arguments as in Section 5.2 show that the chain is regularly varying with index $\alpha>0$. We consider the following function acting on the Markov chain $\left(\Phi_{t}\right)$ :

$$
\mathbf{X}_{t}=f\left(\Phi_{t}\right)=\left(X_{t} \Phi_{t}, X_{t}, \Phi_{t}\right), \quad t \in \mathbb{Z} .
$$

By convention, we will assume that all vectors are understood as column vectors. The sequence $\left(\Phi_{t}\right)$ satisfies the recursion

$$
\Phi_{t}=\left(\begin{array}{ccccc}
A_{t} & 0 & \cdots & 0 & 0 \\
1 & 0 & \cdots & 0 & 0 \\
0 & 1 & \cdots & 0 & 0 \\
\vdots & \vdots & \ddots & \vdots & \vdots \\
0 & 0 & \cdots & 1 & 0
\end{array}\right) \Phi_{t-1}+\left(\begin{array}{c}
B_{t} \\
0 \\
0 \\
\vdots \\
0
\end{array}\right)=\mathbf{A}_{t} \Phi_{t-1}+\mathbf{B}_{t}, \quad t \in \mathbb{Z} .
$$

We will show that $\left(\mathbf{X}_{t}\right)$ satisfies $\left(\mathbf{R V}_{\alpha / 2}\right)$ and $\left(\mathbf{D C}_{p, m}\right)$ for $m$ sufficiently large, $V(x)=|x|^{p}$ and $p<\alpha / 2$. The condition $\mathbb{E}\left(V\left(f\left(\Phi_{1}\right)\right) \mid \Phi_{0}=y\right) \leqslant c_{1} V(f(y))+c_{2}$ for some positive $c_{1}, c_{2}$ follows immediately from the stochastic recurrence equation

$$
\mathbf{X}_{t}=\left(\begin{array}{ccc}
A_{t} \mathbf{A}_{t} & A_{t} \mathbf{B}_{t} & B_{t} \mathbf{A}_{t} \\
0_{1, h+1} & A_{t} & 0_{1, h+1} \\
0_{h+1, h+1} & 0_{h+1,1} & \mathbf{A}_{t}
\end{array}\right) \mathbf{X}_{t-1}+\left(\begin{array}{c}
B_{t} \mathbf{B}_{t} \\
B_{t} \\
\mathbf{B}_{t}
\end{array}\right)=\mathbf{C}_{t} \mathbf{X}_{t-1}+\mathbf{D}_{t}, \quad t \in \mathbb{Z} .
$$

Condition $\mathbb{E}|\mathbf{D}|^{(\alpha+\varepsilon) / 2}<\infty$ follows by the assumptions. From basic algebra, for $m \geqslant h$ the matrix products $\prod_{t=1}^{m} A_{t} \mathbf{A}_{t}=\prod_{t} \prod_{t=1}^{m} \mathbf{A}_{t}$ can be written as $\prod_{m, h} \mathbf{M}_{h}$, where the $(h+1) \times(h+1)$ matrix 
$\mathbf{M}_{h}$ has zero entries but the first column given by $\left(\Pi_{1, h-1}, \Pi_{2, h-1}, \ldots, 1\right)$. Products of triangular matrices remain triangular and their diagonal is the product of the diagonals. Thus we obtain

$$
\mathbf{C}_{m} \cdots \mathbf{C}_{1}=\left(\begin{array}{ccc}
\Pi_{m, h}^{2} I_{h} & 0_{1, h+1} & 0_{h+1, h+1} \\
0_{1, h+1} & \Pi_{m, h} & 0_{1, h+1} \\
0_{h+1, h+1} & 0_{h+1,1} & \Pi_{m, h} I_{h}
\end{array}\right) \widetilde{\mathbf{C}}_{h}=\widetilde{\mathbf{D}}_{m} \widetilde{\mathbf{C}}_{h},
$$

where $\widetilde{\mathbf{C}}_{h}$ is an upper triangular block matrix depending only on $\left(A_{t}\right)_{1 \leqslant t \leqslant h-1}$. The matrices $\widetilde{\mathbf{D}}_{m}$ and $\widetilde{\mathbf{C}}_{h}$ are independent and for some $c>0$ we have

$$
\begin{aligned}
\mathbb{E}\left\|\widetilde{\mathbf{D}}_{m} \widetilde{\mathbf{C}}_{h}\right\|^{p} & \leqslant \mathbb{E}\left\|\widetilde{\mathbf{D}}_{m}\right\|^{p} \mathbb{E}\left\|\widetilde{\mathbf{C}}_{h}\right\|^{p} \\
& \leqslant c \mathbb{E}\left[\left|A_{m}\right|^{2 p} \cdots\left|A_{h}\right|^{2 p}+\left|A_{m}\right|^{p} \cdots\left|A_{h}\right|^{p}\right] \mathbb{E}\left\|\widetilde{\mathbf{C}}_{h}\right\|^{p} .
\end{aligned}
$$

Since $p<\alpha / 2, \mathbb{E}\left(\left|A_{0}\right|^{2 p}\right)^{m} \rightarrow 0$ and $\mathbb{E}\left(\left|A_{0}\right|^{p}\right)^{m} \rightarrow 0$ as $m \rightarrow \infty$. Thus, for $m$ sufficiently large, $\mathbb{E}\left\|\widetilde{\mathbf{D}}_{m} \widetilde{\mathbf{C}}_{h}\right\|^{p} \leqslant c\left(\mathbb{E}\left(\left|A_{0}\right|^{2 p}\right)^{m}+\mathbb{E}\left(\left|A_{0}\right|^{p}\right)^{m}\right)<$ 1, i.e. condition $\left(\mathbf{D C}_{p, m}\right)$ holds, and Lemma 2.1]applies provided we can also show $\left(\mathbf{R V}_{\alpha / 2}\right)$ for $\left(\mathbf{X}_{t}\right)$. This is our next goal. Since $X_{t}$ and $\Phi_{t}$ are regularly varying with index $\alpha$ we deal with a degenerate case where the limiting measure of regular variation of $\mathbf{X}_{t}$ is concentrated at zero for the last $h+2$ components. Then, in view of the definition of the cluster index, $b$ is the same for $\left(X_{t} \Phi_{t}\right)$ and $\left(\mathbf{X}_{t}\right)$. Therefore we will calculate $b$ for $\left(X_{t} \Phi_{t}\right)$. Abusing notation, we will also use the same notation for the tail process. As in Section 5.2 we obtain by iteration of the stochastic recurrence equation $X_{t}=A_{t} X_{t-1}+B_{t}$,

$$
\begin{aligned}
X_{t} \Phi_{t} & =\Pi_{t-h+1, t}\left(\Pi_{t-h+1, t}, \Pi_{t-h+1, t-1}, \ldots, 1\right)^{\prime} X_{t-h}^{2}+\mathbf{R}_{t}^{(1)} \\
& =\Pi_{1-h, t-h}^{2} \Pi_{t-h+1, t}\left(\Pi_{t-h+1, t}, \Pi_{t-h+1, t-1}, \ldots, 1\right)^{\prime} X_{-h}^{2}+\mathbf{R}_{t}^{(2)}, \\
& =\Pi_{1-h, t}\left(\Pi_{1-h, t}, \Pi_{1-h, t-1}, \ldots, \Pi_{1-h, t-h}\right)^{\prime} X_{-h}^{2}+\mathbf{R}_{t}^{(2)},
\end{aligned}
$$

where $\mathbb{E}\left|\mathbf{R}_{t}^{(i)}\right|^{(\alpha+\varepsilon) / 2}<\infty, i=1,2$. Then for $t \geqslant 0$,

$$
\begin{aligned}
& \left(X_{0} \Phi_{0}, \ldots, X_{t} \Phi_{t}\right) \\
& \quad=\left(\begin{array}{cccc}
\Pi_{1-h, 0}^{2} & \Pi_{1-h, 1}^{2} & \cdots & \Pi_{1-h, t}^{2} \\
\Pi_{1-h, 0} \Pi_{1-h,-1} & \Pi_{1-h, 1} \Pi_{1-h, 0} & \cdots & \Pi_{1-h, t} \Pi_{1-h, t-1} \\
\vdots & \vdots & \ddots & \vdots \\
\Pi_{1-h, 0} & \Pi_{1-h, 1} A_{1-h} & \cdots & \Pi_{1-h, t} \Pi_{1-h, t-h}
\end{array}\right) X_{-h}^{2}+\mathbf{Q}_{t}, \quad t \in \mathbb{Z} .
\end{aligned}
$$

and $\mathbb{E}\left|\mathbf{Q}_{t}\right|^{(\alpha+\varepsilon) / 2}<\infty$. In the remainder of this section we assume that $P(A=0)=0$; the general case can be treated as well but leads to tedious case studies. An application of Corollary 3.2 in Basrak and Segers [6] yields that for continuity sets $M$,

$$
\mathbb{P}\left(x^{-1}\left(X_{0} \Phi_{0}, \ldots, X_{t} \Phi_{t}\right) \in M|| X_{0} \Phi_{0} \mid>x\right) \rightarrow P\left(\left|Y_{0}\right| \mathbf{E}_{t} \in M\right),
$$

where

$$
\mathbf{E}_{t} \stackrel{d}{=} \frac{1}{\left|\Pi_{h}\right| \sqrt{\Pi_{h}^{2}+\Pi_{h-1}^{2}+\cdots+1}}\left(\begin{array}{cccc}
\Pi_{h} \Pi_{h} & \Pi_{h+1} \Pi_{h+1} & \cdots & \Pi_{t+h} \Pi_{t+h} \\
\Pi_{h} \Pi_{h-1} & \Pi_{h+1} \Pi_{h} & \cdots & \Pi_{t+h} \Pi_{t+h-1} \\
\vdots & \vdots & \ddots & \vdots \\
\Pi_{h} & \Pi_{h+1} \Pi_{1} & \cdots & \Pi_{t+h} \Pi_{t}
\end{array}\right)
$$

and $\mathbf{E}_{t}$ is independent of $\left|Y_{0}\right|$. The right-hand side can be identified with $\left(\Theta_{0}, \ldots, \Theta_{t}\right)$.

An application of Theorem 4.1 now yields a stable limit result for the sample autocovariance function of $\left(X_{t}\right)$ : Assume that $\left(a_{n}\right)$ satisfies $n \mathbb{P}\left(\left|X_{0} \Phi_{0}\right|>a_{n}\right) \sim 1$. In view of (5.7) and Breiman's result (see [10]) we also have

$$
n \mathbb{P}\left(\left|X_{0} \Phi_{0}\right|>a_{n}\right) \sim n \mathbb{P}\left(X^{2}>a_{n}\right) \mathbb{E}\left[\left(\left|\Pi_{h}\right| \sqrt{1+\Pi_{1}^{2}+\cdots+\Pi_{h}^{2}}\right)^{\alpha / 2}\right] .
$$


In view of Kesten's result [32], $\mathbb{P}(|X|>x)) \sim c_{0} x^{-\alpha}$. Therefore we can choose

$$
a_{n}=n^{2 / \alpha}\left(c_{0} \mathbb{E}\left[\left(\left|\Pi_{h}\right| \sqrt{1+\Pi_{1}^{2}+\cdots+\Pi_{h}^{2}}\right)^{\alpha / 2}\right]\right)^{2 / \alpha} .
$$

Then we have for $m \geqslant 0, \alpha \in(2,4)$,

$$
\left(a_{n}^{-1} \sum_{t=1}^{n-h}\left(X_{t} X_{t+h}-\mathbb{E}\left(X_{0} X_{h}\right)\right)\right)_{h=0, \ldots, m} \stackrel{d}{\rightarrow} \xi_{\alpha / 2},
$$

and for $\alpha \in(0,2)$,

$$
\left(a_{n}^{-1} \sum_{t=1}^{n-h} X_{t} X_{t+h}\right)_{h=0, \ldots, m} \stackrel{d}{\rightarrow} \xi_{\alpha / 2},
$$

where $\xi_{\alpha / 2}$ is an $\alpha / 2$-stable $\mathbb{R}^{h+1}$-valued random vector whose characteristic function is given in Theorem 4.1 and $\left(\Theta_{t}\right)_{t \geqslant 0}$ is described above. This result was proved in Basrak et al. 4, Theorem 2.13. In the case $\alpha \in(2,4)$ the additional condition $(2.20)$ was needed; the latter condition is hardly verifiable and could be overcome in the present paper by showing condition $\left(\mathbf{D C}_{p}\right)$. Moreover, as in [4] a straightforward application of the continuous mapping theorem yields a corresponding limit result for the sample autocorrelation function; we omit details. The limit laws in Theorem 2.13 of 4 are expressed in terms of the points of the limiting point processes in Theorem 4.2 above, while our limits are expressed in terms of the cluster index $b$. Neither of the representations of the $\alpha$-stable limits are easy due to the complicated dependence structure.

5.4. Sample mean of a GARCH $(1,1)$ process and its volatility, sample covariance function of a $\operatorname{GARCH}(1,1)$ process. We consider a $\operatorname{GARCH}(1,1)$ process $X_{t}=\sigma_{t} Z_{t}$, where $\left(Z_{t}\right)$ is an iid sequence of mean zero unit variance random variables and $\left(\sigma_{t}\right)$ is a sequence of non-negative random variables such that $\sigma_{t}^{2}=\alpha_{0}+\sigma_{t-1}^{2}\left(\alpha_{1} Z_{t-1}^{2}+\beta_{1}\right)$. Here $\alpha_{0}, \alpha_{1}, \beta_{1}$ are positive constants. The latter equation is of Kesten type (5.3) with $A_{t}=\alpha_{1} Z_{t-1}^{2}+\beta_{1}$ and $B_{t}=\alpha_{0}$. We assume that the conditions of Section 5.2 are satisfied, in particular,

$$
\mathbb{P}(\sigma>x) \sim c_{0} x^{-\alpha}, \quad x \rightarrow \infty,
$$

for some constant $c_{0}>0$ and tail index $\alpha>0$, satisfying $\mathbb{E}\left(\alpha_{1} Z_{0}^{2}+\beta_{1}\right)^{\alpha / 2}=1$. We also assume that $\mathbb{E}|Z|^{\alpha+\epsilon}<\infty$ for some $\epsilon>0$. Rewriting (5.5), we have

$$
\left(\sigma_{0}^{2}, \ldots, \sigma_{t}^{2}\right)=\sigma_{0}^{2}\left(1, \Pi_{1}, \ldots, \Pi_{t}\right)+\mathbf{R}_{t},
$$

where $\mathbb{E}\left|\mathbf{R}_{t}\right|^{(\alpha+\epsilon) / 2}<\infty$ and also $\mathbb{E}\left|\Pi_{i}\right|^{(\alpha+\epsilon) / 2}<\infty$ for $i \geqslant 1$. An application of Breiman's multivariate result (see Basrak et al. [3]) shows that for any continuity set $M$ as $x \rightarrow \infty$,

$$
\begin{aligned}
\frac{\mathbb{P}\left(x^{-1}\left(\sigma_{0}, \ldots, \sigma_{t}\right) \in M\right)}{\mathbb{P}(\sigma>x)} & \sim \frac{\mathbb{P}\left(x^{-1} \sigma_{0}\left(1, \Pi_{1}^{0.5}, \ldots, \Pi_{t}^{0.5}\right) \in M\right)}{\mathbb{P}(\sigma>x)} \\
& \rightarrow \int_{0}^{\infty} \alpha y^{-\alpha-1} P\left(y\left(1, \Pi_{1}^{0.5}, \ldots, \Pi_{t}^{0.5}\right) \in M\right) d y .
\end{aligned}
$$

This shows that regular variation of $\left(\sigma_{t}\right)$ with index $\alpha$ follows from the regular variation of $\sigma$. This property is inherited by the sequence $\left(X_{t}\right)$. We observe that as $x \rightarrow \infty$,

$$
\frac{\mathbb{P}\left(\left|\left(X_{0}, \ldots, X_{t}\right)-\sigma_{0}\left(Z_{0}, \Pi_{1}^{0.5} Z_{1}, \ldots, \Pi_{t}^{0.5} Z_{t}\right)\right|>x\right)}{\mathbb{P}(\sigma>x)} \leqslant \frac{\mathbb{P}\left(\left|Z_{1}\right| R_{1}^{0.5}+\cdots+\left|Z_{t}\right| R_{t}^{0.5}>x\right)}{\mathbb{P}(\sigma>x)}=o(1) .
$$

In the last step we used the independence of $Z_{i}$ and $R_{i}$ as well as the moment condition on $Z$. Condition $\left(\mathbf{R V}_{\alpha}\right)$ for $\left(X_{t}\right)$ now follows. This property was proved in Mikosch and Stărică [37] under the additional condition that $Z$ be symmetric. The above calculation shows that this assumption can be avoided. 
Next we consider the 2-dimensional Markov chain

$$
\Phi_{t}=\left(\sigma_{t}, X_{t}\right)^{\prime}=\sigma_{t}\left(1, Z_{t}\right)^{\prime}, \quad t \in \mathbb{Z} .
$$

A similar calculation as above shows that this Markov chain satisfies $\left(\mathbf{R V}_{\alpha}\right)$ and for $h \geqslant 0$, any continuity set $N$, observing that $\left|\Phi_{0}\right|=\sigma_{0} \sqrt{Z_{0}^{2}+1}$,

$$
\begin{aligned}
\mathbb{P} & \left(x^{-1}\left(\Phi_{0}, \ldots, \Phi_{h}\right) \in N|| \Phi_{0} \mid>x\right) \\
& \sim \mathbb{P}\left(x^{-1} \sigma_{0}\left(\left(1, Z_{0}\right)^{\prime}, \Pi_{1}^{0.5}\left(1, Z_{1}\right)^{\prime}, \ldots, \Pi_{h}^{0.5}\left(1, Z_{h}\right)^{\prime}\right) \in N|| \Phi_{0} \mid>x\right) \\
& \stackrel{w}{\rightarrow} \mathbb{P}\left(\left|Y_{0}\right|\left(\left(1, Z_{0}\right)^{\prime}, \Pi_{1}^{0.5}\left(1, Z_{1}\right)^{\prime}, \ldots, \Pi_{h}^{0.5}\left(1, Z_{h}\right)^{\prime}\right) /\left(Z_{0}^{2}+1\right)^{0.5} \in N\right) .
\end{aligned}
$$

Identifying the limiting vector with $\left|Y_{0}\right|\left(\Theta_{0}, \ldots, \Theta_{h}\right)^{\prime}$, we have for any $\theta \in \mathbb{S}$,

$$
b(\theta)=\mathbb{E}\left[\left\{\left(\theta^{\prime}\left(1, Z_{0}\right)^{\prime}+\sum_{t \geqslant 1} \Pi_{t}^{0.5} \theta^{\prime}\left(1, Z_{t}\right)^{\prime}\right)_{+}^{\alpha}-\left(\sum_{t \geqslant 1} \Pi_{t}^{0.5} \theta^{\prime}\left(1, Z_{t}\right)^{\prime}\right)_{+}^{\alpha}\right\} /\left(Z_{0}^{2}+1\right)^{\alpha / 2}\right] .
$$

The Markov chain $\left(\Phi_{t}\right)$ is aperiodic and irreducible under classical conditions on the density of the $Z$; see e.g. [37] for details. The condition $\left(\mathbf{D C}_{p}\right)$ for $p<\alpha$ follows by an application of Lemma 2.1 for $V(x)=|x|^{p}$. We recall that for $m \geqslant 2, \sigma_{m}^{2}=\Pi_{2, m}\left(\alpha_{0}+\alpha_{1} X_{0}^{2}+\beta_{1} \sigma_{0}^{2}\right)+\widetilde{R}_{m}$, where $\mathbb{E}\left|\widetilde{R}_{m}\right|^{(\alpha+\epsilon) / 2}<\infty$ for some $\epsilon>0$ and $\widetilde{R}_{m}$ is independent of $Z_{m}$. We have for $p<\alpha$, some $c>0$,

$$
\begin{aligned}
\mathbb{E}\left[\left|\Phi_{m}\right|^{p} \mid \Phi_{0}=\mathbf{y}\right] & =\mathbb{E}\left|\Pi_{2, m}\left(\alpha_{0}+\alpha_{1} y_{1}^{2}+\beta_{1} y_{2}^{2}\right)+\widetilde{R}_{m}\right|^{p / 2} \mathbb{E}\left(Z^{2}+1\right)^{p / 2} \\
& \leqslant|\mathbf{y}|^{p} \mathbb{E}\left|\Pi_{2, m}\right|^{p / 2} \mathbb{E}\left(Z^{2}+1\right)^{p / 2} \max \left(\alpha_{1}^{p}, \beta_{1}^{p}\right)+c .
\end{aligned}
$$

For $m=1$, we find constants $c_{1}, c_{2}>0$ such that $\mathbb{E}\left(V\left(\Phi_{1}\right) \mid \Phi_{0}=\mathbf{y}\right) \leqslant c_{1} V(\mathbf{y})+c_{2}$. Since $\mathbb{E} A^{p / 2}<1$ for $p<\alpha,\left(\mathbf{D C}_{p, m}\right)$ holds for sufficiently large $m$ in view of (5.8). An application of Lemma 2.1 concludes the proof. Thus we may apply the stable limit theory of Theorem 4.1 with $f(x)=x$ to $\left(\Phi_{t}\right)$ for $\alpha<2$ and the limit law is determined by the cluster index $b$ above.

For $h \geqslant 0$ consider the Markov chain, recycling the notation $\Phi_{t}$,

$$
\Phi_{t}=\left(X_{t}, \sigma_{t}, \ldots, X_{t-h}, \sigma_{t-h}\right), \quad t \in \mathbb{Z} .
$$

We also write

$$
\Phi_{t}^{2}=\left(X_{t}^{2}, \sigma_{t}^{2}, \ldots, X_{t-h}^{2}, \sigma_{t-h}^{2}\right), \quad t \in \mathbb{Z},
$$

and introduce the function $f$ acting on $\left(\Phi_{t}\right)$ given by

$$
\mathbf{Y}_{t}=f\left(\Phi_{t}\right)=\left(X_{t}\left(X_{t-1}, \ldots, X_{t-h}\right), \Phi_{t}^{2}, \Phi_{t}\right), \quad t \in \mathbb{Z} .
$$

We intend to show $\left(\mathbf{D C}_{p, m}\right)$ for $p<\alpha / 2$ and some large $m$. We restrict ourselves to the case $h=1$; the general case is analogous but requires more accounting. We observe that for suitable constants $c>0$,

$$
\begin{aligned}
\left|f\left(\Phi_{t}\right)\right|^{p} & =\left|X_{t}^{2} X_{t-1}^{2}+X_{t}^{4}+X_{t-1}^{4}+\sigma_{t}^{4}+\sigma_{t-1}^{4}+X_{t}^{2}+X_{t-1}^{2}+\sigma_{t}^{2}+\sigma_{t-1}^{2}\right|^{p / 2} \\
& \leqslant c\left(\left(1+Z_{t}^{4}\right)\left(1+X_{t-1}^{4}+\sigma_{t-1}^{4}\right)+Z_{t}^{2}\left(1+\sigma_{t-1}^{2}+X_{t-1}^{2}\right)\left(1+X_{t-1}^{2}\right)+X_{t-1}^{2}+1+\sigma_{t-1}^{2}\right)^{p / 2}
\end{aligned}
$$

Then for suitable constants $c_{1}, c_{2}>0$,

$$
\begin{aligned}
\mathbb{E}\left[\left|f\left(\Phi_{1}\right)\right|^{p} \mid \Phi_{0}=\mathbf{y}\right] & \left.\leqslant c\left(1+\left|y_{1}^{2}\right|^{p}+\left|y_{2}^{2}\right|^{p}+\left|y_{1}\right|^{p}+\left|y_{2}\right|^{p}\right)\right) \\
& \leqslant c_{1}|f(\mathbf{y})|^{p}+c_{2} .
\end{aligned}
$$


By a similar argument, for sufficiently large $m \geqslant 1$, suitable constants $c>0$, recalling that $\sigma_{t}^{2}=$ $\Pi_{t} \sigma_{0}^{2}+R_{t}$, where $\sigma_{0}^{2}$ is independent of $\left(\Pi_{t}, R_{t}\right)$, and

$$
\begin{aligned}
\mathbb{E}\left[\left|f\left(\Phi_{m}\right)\right|^{p} \mid \Phi_{0}=\mathbf{y}\right] & \leqslant c\left(1+\mathbb{E}\left[\left|\sigma_{m-1}^{4}\right|^{p / 2}+\left|\sigma_{m-1}^{2}\right|^{p / 2} \mid \Phi_{0}=\mathbf{y}\right]\right) \\
& \left.\leqslant c\left(1+\mathbb{E} \Pi_{m}^{2 p}\left|y_{2}\right|^{2 p}+\mathbb{E} \Pi_{m}^{p}\right]\left|y_{2}\right|^{p}\right) \\
& \leqslant c\left(\mathbb{E}\left[\Pi_{m}^{2 p}\right]+\mathbb{E}\left[\Pi_{m}^{p}\right]\right)|f(\mathbf{y})|^{p}+c \\
& \leqslant \beta|f(\mathbf{y})|^{p}+c,
\end{aligned}
$$

for some $\beta \in(0,1)$, sufficiently large $m \geqslant 1$. Here we used the fact that $\mathbb{E} A^{2 p}<1$ for $p<\alpha / 2$. Now we can apply Lemma 2.1 to show $\left(\mathbf{D C}_{p}\right)$ for $p<\alpha / 2$

It remains to show $\left(\mathbf{R} \mathbf{V}_{\alpha / 2}\right)$ for $\left(\mathbf{Y}_{t}\right)$ defined in (5.9). The $\Phi_{t}$-component of $\mathbf{Y}_{t}$ is regularly varying with index $\alpha$. Therefore, without loss of generality and abusing notation, we will consider the sequence

$$
\mathbf{Y}_{t}=f\left(\Phi_{t}\right)=\left(X_{t}\left(X_{t-1}, \ldots, X_{t-h}\right), \Phi_{t}^{2}\right), \quad t \in \mathbb{Z}
$$

Similar arguments as in the first part of this subsection and as in Section 5.2 show for $t \geqslant 0$ that

$$
\begin{aligned}
& \mathbf{Y}_{t}=\mathbf{R}_{t}^{(1)}+ \\
& \sigma_{t-h}^{2}\left(Z_{t} \Pi_{t-h+1, t}^{0.5}\left(Z_{t-1} \Pi_{t-h+1, t-1}^{0.5}, \ldots, Z_{t-h}\right),\left(\Pi_{t-h+1, t}\left(Z_{t}^{2}, 1\right), \ldots,\left(Z_{t-h}^{2}, 1\right)\right)\right)^{\prime} \\
& =\mathbf{R}_{t}^{(2)}+ \\
& \sigma_{-h}^{2} \Pi_{1-h, t-h}\left(Z_{t} \Pi_{t-h+1, t}^{0.5}\left(Z_{t-1} \Pi_{t-h+1, t-1}^{0.5}, \ldots, Z_{t-h}\right),\left(\Pi_{t-h+1, t}\left(Z_{t}^{2}, 1\right), \ldots,\left(Z_{t-h}^{2}, 1\right)\right)\right)^{\prime},
\end{aligned}
$$

where $\mathbb{E}\left|\mathbf{R}_{t}^{(i)}\right|^{(\alpha+\varepsilon) / 2}<\infty, i=1,2$. Therefore

$$
\left(\mathbf{Y}_{0}, \ldots, \mathbf{Y}_{t}\right)^{\prime}=\widetilde{\mathbf{D}}_{t} \sigma_{-h}^{2}+\widetilde{\mathbf{Q}}_{t}
$$

where $\mathbb{E}\left|\widetilde{\mathbf{Q}}_{t}\right|^{(\alpha+\varepsilon) / 2}<\infty$ and $\mathbb{E}\left|\widetilde{\mathbf{D}}_{t}\right|^{(\alpha+\varepsilon) / 2}<\infty$ for some $\varepsilon>0$ and

$\widetilde{\mathbf{D}}_{t}=\left(\begin{array}{cccc}Z_{0} Z_{-1} \Pi_{1-h, 0}^{0.5} \Pi_{1-h}^{0.5} & Z_{1} Z_{0} A_{1-h} \Pi_{2-h, 1}^{0.5} \Pi_{2-h, 0}^{0.5} & \cdots & Z_{t} Z_{t-1} \Pi_{1-h, t-h} \Pi_{t-h+1, t}^{0.5} \Pi_{t-h+1, t-1}^{0.5} \\ Z_{0} Z_{-2} \Pi_{1-h, 0}^{0.5} \Pi_{1-h,-2}^{0.5} & Z_{1} Z_{-1} A_{1-h} \Pi_{2-h, 1}^{0.5} \Pi_{2-h,-1}^{0.5} & \cdots & Z_{t} Z_{t-2} \Pi_{1-h, t-h} \Pi_{t-h+1, t}^{0.5} \Pi_{t-h+1, t-2}^{0.5} \\ \vdots & \vdots & \ddots & \vdots \\ Z_{0} Z_{-h} \Pi_{1-h, 0}^{0.5} & Z_{1} Z_{1-h} A_{1-h} \Pi_{2-h, 1}^{0.5} & \cdots & Z_{t} Z_{t-h} \Pi_{1-h, t-h} \Pi_{t-h+1, t}^{0.5} \\ \Pi_{1-h, 0}\left(Z_{0}^{2}, 1\right) & A_{1-h} \Pi_{2-h, 1}\left(Z_{1}^{2}, 1\right) & \cdots & \Pi_{1-h, t-h} \Pi_{t-h+1, t}\left(Z_{t}^{2}, 1\right) \\ \Pi_{1-h,-1}\left(Z_{-1}, 1\right) & A_{1-h} \Pi_{2-h, 0}\left(Z_{0}^{2}, 1\right) & \cdots & \Pi_{1-h, t-h} \Pi_{t-h+1, t-1}\left(Z_{t-1}^{2}, 1\right) \\ \vdots & \vdots & \ddots & \vdots \\ \left(Z_{-h}^{2}, 1\right) & A_{1-h}\left(Z_{1-h}^{2}, 1\right) & \cdots & \Pi_{1-h, t-h}\left(Z_{t-h}^{2}, 1\right)\end{array}\right)$.

Notice that $\sigma_{-h}^{2}$ and $\widetilde{\mathbf{D}}_{t}$ are independent and that $\sigma_{-h}^{2}$ is regularly varying with index $\alpha / 2$. Then $\left(\mathbf{R V}_{\alpha / 2}\right)$ for $\left(\mathbf{Y}_{0}, \ldots, \mathbf{Y}_{t}\right)$ follows by an application of the multivariate Breiman result; see [3]. We omit the calculation of the cluster index; it is similar to its calculation in Section 5.2

Now we can apply Theorem 4.1 to prove limit theory with $\alpha / 2$-stable limits, $\alpha<4$, for the sample autocovariance function of the $\operatorname{GARCH}(1,1)$ process. The corresponding theory using point process techniques is given in [17, 37]. There the limit theory for the sequences $\left(\left|X_{t}\right|\right)$ and $\left(X_{t}^{2}\right)$ was also provided. The same results can be provided by Theorem 4.1 by calculating the corresponding cluster indices. Applied to the squares $\left(X_{t}^{2}\right)$ we obtain in particular for $\alpha \in(2,4)$,

$$
n a_{n}^{-1} \frac{1}{n} \sum_{t=1}^{n-h} X_{t}^{2} X_{t+h}^{2}-\left(\frac{1}{n} \sum_{t=1}^{n} X_{t}^{2}\right)^{2} \stackrel{d}{\rightarrow} \xi_{\alpha / 4}
$$


where $\xi_{\alpha / 4}$ is an $\alpha / 4$-stable random variable whose characteristic function is given in Theorem 4.1 and $\left(\Theta_{t}\right)_{t \geqslant 0}=\left(c Z_{t}^{2} Z_{t+h}^{2} \Pi_{t} \Pi_{t+h}\right)_{t \geqslant 0}$ for some $c>0$. In particular, the $\Theta_{t}$ s are non negative and thus $b_{-}=0$. Then $\xi_{\alpha / 4}$ is supported on $\left[-\left(\mathbb{E} X_{0}^{2}\right)^{2}, \infty\right)$. We omit further details. Relation (5.10) supports the idea of spurious long-range dependence effects observed on real-life log-return data which are often observed to have infinite fourth moments; see 38 for a discussion.

\section{Proof of Theorem 4.1}

6.1. Proof of part (1). We will use the Cramér-Wold device to show that $\left(a_{n}^{-1} \theta^{\prime} S_{n}\right)$ has a (possibly degenerate) $\alpha$-stable limit $\xi_{\alpha}(\theta)$ for every $\theta \in \mathbb{S}^{d-1}$. We will apply Theorem 1 in 2 which we recall for convenience:

Theorem 6.1. Assume that $\left(G_{t}\right)$ is a strictly stationary process of random variables, satisfying the following conditions.

(1) The regular variation condition $\left(\mathbf{R V}_{\alpha}\right)$ holds for some $\alpha \in(0,2)$.

(2) The mixing condition (MX): There exist $m=m_{n} \rightarrow \infty$ such that $k_{n}=\left[n / m_{n}\right] \rightarrow \infty$ and

$$
\mathbb{E} \mathrm{e}^{i t b_{n}^{-1} S_{n}(G)}-\left(\mathbb{E} \mathrm{e}^{i t b_{n}^{-1} S_{m}(G)}\right)^{k_{n}} \rightarrow 0, \quad n \rightarrow \infty, \quad t \in \mathbb{R},
$$

where $S_{n}(G)=G_{1}+\cdots+G_{n}$ and $\left(b_{n}\right)$ is chosen such that $n \mathbb{P}\left(\left|G_{1}\right|>b_{n}\right) \sim 1$.

(3) The anti-clustering condition

$(\mathrm{AC})$

$$
\lim _{\ell \rightarrow \infty} \limsup _{n \rightarrow \infty} \frac{n}{m} \sum_{j=\ell+1}^{m} \mathbb{E}\left|\overline{x b_{n}^{-1}\left(S_{j}(G)-S_{\ell}(G)\right)} \overline{x b_{n}^{-1} G_{1}}\right|=0, \quad x \in \mathbb{R},
$$

holds, where $m=m_{n}$ is the same as in $(\mathbf{M X})$ and $\bar{x}=(x \wedge 2) \vee(-2)$.

(4) The limits

$$
\lim _{\ell \rightarrow \infty}\left(b_{+}(\ell)-b_{+}(\ell-1)\right)=c_{+} \text {and } \lim _{\ell \rightarrow \infty}\left(b_{-}(\ell)-b_{-}(\ell-1)\right)=c_{-},
$$

exist. Here $b_{+}(\ell), b_{-}(\ell)$ are the tail balance parameters given by $b_{+}(\ell)=\lim _{n \rightarrow \infty} n P\left(S_{\ell}(G)>\right.$ $\left.b_{n}\right)$ and $b_{-}(\ell)=\lim _{n \rightarrow \infty} n P\left(S_{\ell}(G) \leqslant-b_{n}\right)$.

(5) For $\alpha>1$ assume $\mathbb{E} G_{1}=0$ and for $\alpha=1$,

$$
\lim _{\ell \rightarrow \infty} \limsup _{n \rightarrow \infty} n\left|\mathbb{E}\left(\sin \left(b_{n}^{-1} S_{\ell}(G)\right)\right)\right|=0 .
$$

Then $c_{+}$and $c_{-}$are non-negative and $\left(b_{n}^{-1} S_{n}(G)\right)$ converges in distribution to an $\alpha$-stable random variable (possibly zero) with characteristic function $\psi_{\alpha}(x)=\exp \left(-|x|^{\alpha} \chi_{\alpha}\left(x, c_{+}, c_{-}\right)\right)$, where for $\alpha \neq 1$ the function $\chi_{\alpha}\left(x, c_{+}, c_{-}\right), x \in \mathbb{R}$, is given by the formula

$$
\frac{\Gamma(2-\alpha)}{1-\alpha}\left(\left(c_{+}+c_{-}\right) \cos (\pi \alpha / 2)-i \operatorname{sign}(x)\left(c_{+}-c_{-}\right) \sin (\pi \alpha / 2)\right),
$$

while for $\alpha=1$ one has

$$
\chi_{1}\left(x, c_{+}, c_{-}\right)=0.5 \pi\left(c_{+}+c_{-}\right)+i \operatorname{sign}(x)\left(c_{+}-c_{-}\right) \log |x|, \quad x \in \mathbb{R} .
$$

We will verify the conditions of this theorem for the sequence $G_{i}=\theta^{\prime} X_{i}$ for fixed $\theta \in \mathbb{S}^{d-1}$.

The regular variation condition $\left(\mathbf{R V}_{\alpha}\right)$ for $\left(G_{t}\right)$. This condition is straightforward from the definition of $\left(\mathbf{R V}_{\alpha}\right)$ for $\left(X_{t}\right)$ and the fact that the function $f(x)=\theta^{\prime} x, x \in \mathbb{R}^{d}$, is continuous and homogeneous. 
The anti-clustering condition (AC). Without loss of generality we assume that $\left(\mathbf{D C}_{p}\right)$ holds for $V(y)=|y|^{p}$. We also assume $p \leqslant 1$; for $p>1$ an application of Jensen's inequality yields $\left(\mathbf{D C}_{p^{\prime}}\right)$ for any $p^{\prime}<p$. Since $p \leqslant 1$ there exists $c>0$ such that $y \leqslant c y^{p}$ for $y \in[0,2]$. Then one has

$$
\begin{aligned}
T_{\ell m} & =\frac{n}{m} \sum_{j=\ell+1}^{m} \mathbb{E}\left[\overline{\left|x b_{n}^{-1}\left(S_{j}(G)-S_{\ell}(G)\right)\right|} \overline{\left|x b_{n}^{-1} G_{1}\right|}\right] \\
\leqslant & \leqslant \frac{n}{m} \sum_{j=\ell+1}^{m} \mathbb{E}\left[\overline{\left|x b_{n}^{-1}\left(S_{j}(G)-S_{\ell}(G)\right)\right|^{p}} \overline{\left|x b_{n}^{-1} G_{1}\right|}\right] .
\end{aligned}
$$

Using $\left(\mathbf{D C}_{p}\right)$, a recursive argument yields

$$
\mathbb{E}\left(\left|G_{k}\right|^{p} \mid \Phi_{1}=y\right) \leqslant \beta^{k-1}|f(y)|^{p}+b \sum_{j=1}^{k-1} \beta^{j}, \quad k \geqslant 2,
$$

where $\beta, b$ appear in $\left(\mathbf{D C}_{p}\right)$. Multiple use of this argument and the subadditivity of the function $z \mapsto \bar{z}$ on $(0, \infty)$ yield for $\ell<j \leqslant m$,

$$
\mathbb{E}\left[\overline{\left|x b_{n}^{-1}\left(S_{j}(G)-S_{\ell}(G)\right)\right|^{p}} \mid \Phi_{1}\right] \leqslant c \overline{|x|^{p} b_{n}^{-p} \sum_{i=\ell+1}^{m} \beta^{i}\left|X_{1}\right|^{p}}+c b_{n}^{-p} m .
$$

Conditioning on $\Phi_{1}$, the latter inequality finally yields

$$
\mathbb{E} T_{\ell m} \leqslant c \frac{n}{m} \sum_{j=\ell+1}^{m} \mathbb{E}\left[\overline{\left[|x|^{p} b_{n}^{-p} \sum_{i=1}^{j} \beta^{i}\left|X_{1}\right|^{p}\right.} \overline{x b_{n}^{-1}\left|X_{1}\right|}\right]+c \frac{m n}{b_{n}^{p}} \overline{\mathbb{E}} \overline{\left|x b_{n}^{-1} X_{1}\right|}=I_{1}+I_{2} .
$$

We have $I_{2} \leqslant c b_{n}^{-p-1} n m=o(1)$ if we choose $m=m_{n}=\log ^{2} n$. It remains to prove that $I_{1}$ is asymptotically negligible. An application of Karamata's theorem yields the bound

$$
I_{1} \leqslant c \frac{n}{m} \sum_{j=\ell+1}^{m} \mathbb{P}\left(\left|X_{1}\right| \geqslant c b_{n}\left(\sum_{i=\ell}^{j} \beta^{i}\right)^{-1 /(p+1)}\right) \leqslant \frac{c}{m} \sum_{j=\ell+1}^{m}\left(\sum_{i=\ell}^{j} \beta^{i}\right)^{\alpha /(p+1)} \leqslant c \beta^{\ell \alpha /(p+1)} .
$$

The right-hand side vanishes as $\ell \rightarrow \infty$. Collecting the above bounds, condition (AC) follows.

The mixing condition (MX). Here we give a significant improvement on Lemma 3 in [2]; in the latter paper it is assumed that $\left(G_{t}\right)$ is strongly mixing. The next result avoids this condition.

Lemma 6.2. Consider a strictly stationary real-valued sequence $\left(G_{t}\right)$ satisfying the conditions $\left(\mathbf{R V}_{\alpha}\right)$ for some $\alpha \in(0,2)$ and $(\mathbf{A C})$. Then $(\mathbf{M X})$ can be replaced by Condition (MX'): There exists a sequence $\left(r_{n}\right)$ such that $r_{n}=o\left(m_{n}\right)$ and

$$
\left|\varphi_{n}^{(\ell)}(t)-\varphi_{n, m-\ell}^{k}(t)\right| \rightarrow 0, \quad t \in \mathbb{R} .
$$

holds for $\ell=m-r_{n}$ and $\ell=r_{n}$, where

$$
\begin{aligned}
\varphi_{n}^{(\ell)}(t) & =\mathbb{E}\left[\exp \left(i t b_{n}^{-1} \sum_{i=1}^{k_{n}} \sum_{t=(i-1) m+1}^{i m-\ell} G_{t}\right)\right] \\
\varphi_{n, j} & =\mathbb{E}\left[\exp \left(i t b_{n}^{-1} \sum_{t=1}^{j} G_{t}\right)\right], \quad j \geqslant 1, \quad \varphi_{n}(t)=\varphi_{n, n}(t), \quad t \in \mathbb{R} .
\end{aligned}
$$


Proof. Notice that condition (MX) can be written in the form $\varphi_{n}(t)-\varphi_{n, m}^{k}(t) \rightarrow 0$ as $n \rightarrow \infty$. We have

$$
\begin{aligned}
\varphi_{n}(t)-\varphi_{n, m}^{k}(t) & =\left[\varphi_{n}(t)-\varphi_{n}^{(r)}(t)\right]+\left[\varphi_{n}^{(r)}(t)-\varphi_{n, m-r}^{k}(t)\right]+\left[\varphi_{n, m-r}^{k}(t)-\varphi_{n, m}^{k}(t)\right] \\
& =P_{1}+P_{2}+P_{3} .
\end{aligned}
$$

In view of (MX)', $P_{2} \rightarrow 0$. Next we deal with $P_{1}$. Assume for simplicity that $k_{n}=n / m$ is an integer. We use the classical Bernstein blocks technique, writing

$$
S_{n}=b_{n}^{-1} \sum_{i=1}^{k_{n}} \sum_{t=(i-1) m+1}^{i m-r} G_{t}+b_{n}^{-1} \sum_{i=1}^{k_{n}} \sum_{t=i m-r+1}^{i m} G_{t}=I_{1}+I_{2} .
$$

We will show that $\mathbb{E} \exp \left(i t I_{2}\right) \rightarrow 1$. Condition (MX)' implies that $\left|\mathbb{E} \exp \left(i t I_{2}\right)-\varphi_{n, r}^{k}(t)\right| \rightarrow 0$ as $\ell=m-r \geqslant r$ and $\ell / n \rightarrow 0$. Moreover, Lemma 3.5 in 44] yields that $\varphi_{n, r}^{k}(t) \rightarrow 1$ if and only if $k\left(\varphi_{n, r}(t)-1\right) \rightarrow 0$. Assuming $\left(\mathbf{R V}_{\alpha}\right)$ and $(\mathbf{A C})$, one can follow the proof of Lemma 1 in [2]. We have

$$
\lim _{q \rightarrow \infty} \limsup _{n \rightarrow \infty}\left|k\left(\varphi_{n, r}(t)-1\right)-k r\left(\varphi_{n, q}(t)-\varphi_{n, q-1}(t)\right)\right| \rightarrow 0, \quad t \in \mathbb{R} .
$$

Under $\left(\mathbf{R V}_{\alpha}\right)$, an application of Theorem 3 in Section XVII.5 of Feller gives that $n\left(\varphi_{n, q}(t)-1\right)$ converges for all $q$. We deduce that $n\left(\varphi_{n, q}(t)-\varphi_{n, q-1}(t)\right)$ converges too. As $k r / n \sim r / m \rightarrow 0$ we conclude that $\operatorname{kr}\left(\varphi_{n, q}(t)-\varphi_{n, q-1}(t)\right) \rightarrow 0$ and then $k_{n}\left(\varphi_{n, r}(t)-1\right) \rightarrow 0$ which gives the desired result $\mathbb{E} \exp \left(i t I_{2}\right) \rightarrow 1$, equivalently, $I_{2} \stackrel{P}{\rightarrow} 0$. Since

$$
\left|P_{1}\right|=\left|\mathbb{E}\left[\exp \left(i t\left(I_{1}\right)\left(1-\exp \left(i t I_{2}\right)\right)\right)\right]\right| \leqslant \mathbb{E}\left|1-\exp \left(i t I_{2}\right)\right|
$$

dominated convergence yields $P_{1} \rightarrow 0$. Finally,

$$
\left|P_{3}\right| \leqslant k\left|\left(\varphi_{n, m-r}(t)-1\right)-\left(\varphi_{n, m}(t)-1\right)\right| \rightarrow 0 .
$$

and the same arguments as above show that $P_{3} \rightarrow 0$.

We finish the proof of $(\mathbf{M X})$ for the sequence $\left(G_{t}\right)$. In view of $\left(\mathbf{D C}_{p}\right),\left(X_{t}\right)$, hence $\left(G_{t}\right)$, are $\beta$-mixing, hence strongly mixing, with exponential rate $\left(\alpha_{h}\right)$. We will show (MX) by an application of Lemma 6.2. A standard telescoping sum argument shows that

$$
\left|\varphi_{n}^{(\ell)}(t)-\varphi_{n, m-\ell}^{k}(t)\right| \leqslant c k_{n} \alpha_{\ell} .
$$

Since we choose $m=\log ^{2} n$ in the proof of (AC), $k_{n} \alpha_{\ell} \leqslant\left(n / \log ^{2} n\right) \exp \left(-c \ell_{n}\right)$. Thus, choosing $\ell_{n}=C \log n$ for some sufficiently large constant $C>0$ we have $\ell_{n}=o\left(m_{n}\right), k_{n} \alpha_{\ell}=o(1)$ and we can also find $r_{n}=o\left(\ell_{n}\right)$. This proves (MX'), hence (MX).

Condition (TB). Note that $\left\{\left|\theta^{\prime} X\right|>b_{n}\right\} \subset\left\{|X|>b_{n}\right\}$. Then

$$
\begin{aligned}
b_{+}(\ell) & =\lim _{x \rightarrow \infty} \frac{\mathbb{P}\left(S_{\ell}(G)>x\right)}{\mathbb{P}\left(\left|\theta^{\prime} X\right|>x\right)} \\
& =\lim _{x \rightarrow \infty} \frac{\mathbb{P}\left(\theta^{\prime} S_{\ell}>x\right)}{\mathbb{P}(|X|>x)} \lim _{x \rightarrow \infty} \frac{\mathbb{P}(|X|>x)}{\mathbb{P}\left(\left|\theta^{\prime} X\right|>x\right)} \\
& =b_{\ell}(\theta) \lim _{x \rightarrow \infty}\left(\mathbb{P}\left(\left|\theta^{\prime} X\right|>x|| X \mid>x\right)\right)^{-1} \\
& \left.=b_{\ell}(\theta)\left(\mathbb{P}\left(\left|Y_{0}\right|\left|\theta^{\prime} \Theta_{0}\right|>1\right)\right)\right)^{-1} \\
& =b_{\ell}(\theta)\left(\mathbb{E}\left(\left|\theta^{\prime} \Theta_{0}\right|^{\alpha}\right)\right)^{-1} .
\end{aligned}
$$

Correspondingly, $b_{-}(\ell)=b_{\ell}(-\theta)\left(\mathbb{E}\left(\left|\theta^{\prime} \Theta_{0}\right|^{\alpha}\right)\right)^{-1}$. Here we assumed that $\mathbb{E}\left(\left|\theta^{\prime} \Theta_{0}\right|^{\alpha}\right) \neq 0$. Otherwise, $b_{+}(\ell)=b_{-}(\ell)=0$. 
Thus we may apply Theorem 6.1 to conclude that $b_{n}^{-1} \theta^{\prime} S_{n} \stackrel{d}{\rightarrow} \xi_{\alpha}(\theta)$ for an $\alpha$-stable random variable $\xi_{\alpha}(\theta)$ with characteristic function $\psi_{\alpha}(x, \theta)$ given by

$$
\begin{aligned}
& \mathbb{E}\left(\left|\theta^{\prime} \Theta_{0}\right|^{\alpha}\right) \log \psi_{\alpha}(x, \theta) \\
& \quad=-|x|^{\alpha} \frac{\Gamma(2-\alpha)}{1-\alpha}((b(\theta)+b(-\theta)) \cos (\pi \alpha / 2)-i \operatorname{sign}(x)(b(\theta)-b(-\theta)) \sin (\pi \alpha / 2)), \quad x \in \mathbb{R} .
\end{aligned}
$$

The factor $\mathbb{E}\left(\left|\theta^{\prime} \Theta_{0}\right|^{\alpha}\right)$ on the left-hand side is due to the normalization $\left(b_{n}\right)$ instead of $\left(a_{n}\right)$. Replacing $\left(b_{n}\right)$ by $\left(a_{n}\right)$, we have for any $v \in \mathbb{R}^{d}$ that

$$
\begin{aligned}
& \mathbb{E} \mathrm{e}^{i v^{\prime}\left(a_{n}^{-1} S_{n}\right)} \rightarrow \\
& \exp \left\{-|v|^{\alpha} C_{\alpha}^{-1}((b(v /|v|)+b(-v /|v|))-i(b(v /|v|)-b(-v /|v|)) \tan (\pi \alpha / 2))\right\},
\end{aligned}
$$

where $C_{\alpha}$ is defined in (4.2). This is the characteristic function of an $\alpha$-stable random vector $\xi_{\alpha}$. The representation of the Lévy spectral measure $\Gamma_{\alpha}$ in (4.1) follows by calculations as in Example 2.3.4 of [50]. Indeed, keeping notations of [50] and identifying the limiting law yields the equations

$b(\theta)+b(-\theta)=C_{\alpha} \sigma_{\theta}^{\alpha}=C_{\alpha} \int_{\mathbb{S}^{d-1}}\left|\theta^{\prime} s\right|^{\alpha} \Gamma_{\alpha}(d s)=C_{\alpha} \int_{\mathbb{S}^{d-1}}\left(\theta^{\prime} s\right)_{+}^{\alpha} \Gamma_{\alpha}(d s)+C_{\alpha} \int_{\mathbb{S}^{d-1}}\left(-\theta^{\prime} s\right)_{+}^{\alpha} \Gamma_{\alpha}(d s)$,

and

$$
\begin{aligned}
b(\theta)-b(-\theta) & =(b(\theta)+b(-\theta)) \beta_{\theta} \\
& =C_{\alpha} \int_{\mathbb{S}^{d-1}}\left|\theta^{\prime} s\right|^{\alpha} \operatorname{sign}\left(\theta^{\prime} s\right) \Gamma_{\alpha}(d s) \\
& =C_{\alpha} \int_{\mathbb{S}^{d-1}}\left(\theta^{\prime} s\right)_{+}^{\alpha} \Gamma_{\alpha}(d s)-C_{\alpha} \int_{\mathbb{S}^{d-1}}\left(-\theta^{\prime} s\right)_{+}^{\alpha} \Gamma_{\alpha}(d s), \quad \theta \in \mathbb{S}^{d-1} .
\end{aligned}
$$

The limiting $\alpha$-stable distribution is degenerate if and only if $b(\theta)=0$ for all $\theta \in \mathbb{S}^{d-1}$.

This proves part (1) of the theorem.

Stable limit theory for general regularly varying stationary processes. In this part we want to give some arguments showing that the results of Theorem 4.1 can be applied in much more general context. For this reason, consider a strictly stationary $\mathbb{R}^{d}$-valued regularly varying sequence $\left(X_{t}\right)$ with index $\alpha>0$. Then $\Phi_{t}=\left(X_{t}, X_{t-1}, \ldots\right), t \in \mathbb{Z}$, constitutes a Markov chain with infinitedimensional state space. In this setting, $\left(\mathbf{D C}_{p}\right)$ for $X_{t}=f\left(\Phi_{t}\right)$ takes on the form:

Condition $\left(\mathbf{D C}_{p}^{\prime}\right)$ :

$$
\mathbb{E}\left(\left|X_{1}\right|^{p}||\left(X_{0}, X_{-1}, \ldots\right)=\left(x_{0}, x_{-1}, \ldots\right)\right) \leqslant \beta\left|x_{0}\right|^{p}+b \quad \text { for some } 0<\beta<1 \text { and } b>0 .
$$

We also need a weak dependence assumption more general than geometric $\beta$-mixing which, in the irreducible case, is implied by the drift condition.

Condition $\left(\mathbf{M X}_{m}\right)$ : Consider an integer sequence $\left(m_{n}\right)$ such that $m=m_{n} \rightarrow \infty$ and $m_{n} / n=o(1)$ and also write $k_{n}=[n / m]$. There exists a sequence $\left(r_{n}\right)$ such that $r_{n}=o\left(m_{n}\right)$ and

$$
\lim _{n \rightarrow \infty}\left|\varphi_{n}^{(\ell)}(s)-\varphi_{n, m-\ell}^{k}(s)\right| \rightarrow 0, \quad s \in \mathbb{R}^{d},
$$

holds for both $\ell=\ell_{n}=m_{n}-r_{n}$ and $\ell=r_{n}$, where

$$
\begin{aligned}
\varphi_{n}^{(\ell)}(s) & =\mathbb{E}\left[\exp \left(i a_{n}^{-1} \sum_{i=1}^{k_{n}} \sum_{t=(i-1) m+1}^{i m-\ell} s^{\prime} X_{t}\right)\right] \\
\varphi_{n, j} & =\mathbb{E}\left[\exp \left(i a_{n}^{-1} \sum_{t=1}^{j} s^{\prime} X_{t}\right)\right], \quad j \geqslant 1, \quad \varphi_{n}(s)=\varphi_{n, n}(s), \quad s \in \mathbb{R}^{d} .
\end{aligned}
$$


Condition $\left(\mathbf{M X}_{m}\right)$ is implied by $\theta$-weak dependence introduced by Doukhan and Louhichi [18: For every $m \geqslant 1$, equip $\left(\mathbb{R}^{d}\right)^{m}$ with the metric $|\cdot|_{m}=m^{-1} \sum_{i=1}^{m}|\cdot|$. A function $f:\left(\mathbb{R}^{d}\right)^{m} \mapsto[-1,1]$, $m \geqslant 1$, is Lipschitz if

$$
\sup _{x \neq y} \frac{|f(x)-f(y)|}{|x-y|_{m}}=\operatorname{Lip}(f)<\infty
$$

The $\theta$-weak dependence coefficients $\left(\theta_{r}\right)_{r \geqslant 0}$ are defined for any $f$ with $\operatorname{Lip}(f)=1$ and measurable $g:\left(\mathbb{R}^{d}\right)^{v} \mapsto[-1,1], v \geqslant 1$, as

$$
\sup _{k, v \geqslant 1} \sup _{i_{1}<\cdots<i_{v} \leqslant 0 \leqslant r \leqslant j_{1}<\cdots<j_{m}}\left|\operatorname{cov}\left(g\left(X_{i_{1}}, \ldots, X_{i_{v}}\right), f\left(X_{j_{1}}, \ldots, X_{j_{m}}\right)\right)\right|=\theta_{r}
$$

Condition ( $\left.\mathbf{M X}_{m}\right)$ follows if $\theta_{r} \rightarrow 0$ for some $r=r_{n}=o(m)$ with $m=m_{n}$. $\theta$-weak dependence covers a wide range of known dependence concepts, including a large variety of mixing conditions; see [18.

In the general case, the following analog of Theorem 4.1 holds. The proof follows along the lines of Theorem 4.1. Irreducibility of $\left(X_{t}\right)$ can be replaced by $\left(\mathbf{M X}_{m}\right)$. We omit further details.

Theorem 6.3. Consider an $\mathbb{R}^{d}$-valued strictly stationary sequence $\left(X_{t}\right)$ satisfying the following conditions:

- $\left(\mathbf{R V}_{\alpha}\right)$ for some $\alpha \in(0,2), \mathbb{E} X=0$ if $\alpha>1$ and $X$ is symmetric if $\alpha=1$.

- $\left(\mathbf{D C}_{p}^{\prime}\right)$ for some $p \in((\alpha-1) \vee 0, \alpha)$.

- $\left(\mathbf{M X}_{m}\right)$ for $m_{n}=o\left(n^{(p+1) / \alpha-1}\right)$.

Let $\left(a_{n}\right)$ be a sequence of positive numbers such that $n \mathbb{P}\left(\left|X_{0}\right|>a_{n}\right) \sim 1$. Then the statement of part (1) of Theorem 4.1 holds.

6.2. Proof of part (2). Recall the regenerative structure of the Markov chain $\left(X_{t}\right)$ from Section 2.2 We will show that the partial sum $S(1)$ over a full regenerative cycle is regularly varying with index $\alpha$. We write

$$
S_{n}=S(0)+\sum_{t=1}^{N_{A}(n)} S(t)+\sum_{i=N_{A}(n)+1}^{n} X_{i}
$$

where $N_{A}(n)=\#\left\{i \geqslant 0: \tau_{A}(i) \leqslant n\right\}, n \geqslant 1$, is independent of $(S(i))_{i \geqslant 1}$. The first and last block sums $S(0)$ and $\sum_{i=\tau_{A}\left(N_{A}(n)\right)+1}^{n} X_{i}$ are tight. Therefore

$$
a_{n}^{-1} S_{n}=a_{n}^{-1} \sum_{t=1}^{N_{A}(n)} S(t)+o_{P}(1) .
$$

By virtue of $\left(\mathbf{D C}_{p}\right)$ for some $p>0$ the chain $\left(X_{t}\right)$ is geometrically ergodic. Therefore there exists a constant $\kappa>0$ such that

$$
\sup _{x \in A} \mathbb{E}_{x} \mathrm{e}^{\kappa \tau_{A}}<\infty,
$$


(see 36], (15.2) in Theorem 15.0.1) and hence $\tau_{A}$ has exponential moment. By a standard renewal argument, $N_{A}(n) / n \stackrel{\text { a.s. }}{\rightarrow}\left(\mathbb{E} \tau_{A}\right)^{-1}$. Then for $\epsilon, \delta>0$,

$$
\begin{aligned}
& \mathbb{P}\left(a_{n}^{-1}\left|\sum_{t=1}^{N_{A}(n)} S(t)-\sum_{t=1}^{n\left(\mathbb{E} \tau_{A}\right)^{-1}} S(t)\right| \geqslant \epsilon\right) \\
& \leqslant \mathbb{P}\left(\left|N_{A}(n)-n\left(\mathbb{E} \tau_{A}\right)^{-1}\right|>\delta N_{A}(n)\right) \\
& \quad+\mathbb{P}\left(a_{n}^{-1}\left|\sum_{t=1}^{\left|N_{A}(n)-n\left(\mathbb{E} \tau_{A}\right)^{-1}\right|} S(t)\right| \geqslant \epsilon,\left|N_{A}(n)-n\left(\mathbb{E} \tau_{A}\right)^{-1}\right| \leqslant \delta N_{A}(n)\right) \\
& \leqslant o(1)+c \mathbb{P}\left(a_{n}^{-1}\left|\sum_{t=1}^{\delta N_{A}(n)} S(t)\right| \geqslant 0.5 \epsilon\right) .
\end{aligned}
$$

In the last step we used a maximal inequality of Ottaviani type; see e.g. [44, Chapter 2. The second term on the right-hand side is neglible, as first letting $n \rightarrow \infty$ and then $\delta \rightarrow 0$ since $a_{n}^{-1} \sum_{t=1}^{N_{A}(n)} S(t) \stackrel{d}{\rightarrow} \xi_{\alpha}$. Hence

$$
a_{n}^{-1} S_{n}=a_{n}^{-1} \sum_{t=1}^{n\left(\mathbb{E} \tau_{A}\right)^{-1}} S(t)+o_{P}(1)
$$

In view of part (1), the sum of iid random vectors $(S(i))$ on the right-hand side has an $\alpha$-stable limit. It follows from [49] that $S(1)$ is regularly varying with index $\alpha$. This concludes the proof.

\section{Proof of Theorem 4.3}

7.1. Proof of part (1): The case $\alpha \in(0,2)$. Recall the decomposition (6.2) of the partial sums $S_{n}$ in terms of the regenerative cycles of the Markov chain. We start with an auxiliary result which deals with the sums over the first and last blocks.

Lemma 7.1. Assume that $\left(\mathbf{R V}_{\alpha}\right)$ and $\left(\mathbf{D C}_{p}\right)$ hold for some $p>\alpha-1$ provided $\alpha>1$. Then there exists a constant $c>0$ such that for any sequence $x=x_{n} \rightarrow \infty$ as $n \rightarrow \infty$,

$$
\begin{aligned}
\mathbb{P}_{A}\left(\sum_{t=1}^{\tau_{A}}\left|X_{t}\right|>x\right) & \leqslant c \mathbb{P}(|X|>x), \\
\mathbb{P}\left(\sum_{t=1}^{\tau_{A}}\left|X_{t}\right|>x, \tau_{A} \leqslant n\right) & =o(n \mathbb{P}(|X|>x)) .
\end{aligned}
$$

Proof. We start by proving (17.1). For any random vector $X$ we write $\bar{X}=X \mathbb{1}_{\{|X| \leqslant x\}}$. Then

$$
\mathbb{P}_{A}\left(\sum_{t=1}^{\tau_{A}}\left|X_{t}\right|>x\right) \leqslant \mathbb{P}_{A}\left(\sum_{t=1}^{\tau_{A}} \overline{\left|X_{t}\right|}>x / 2\right)+\mathbb{P}_{A}\left(\cup_{t=1}^{\tau_{A}}\left\{\overline{\left|X_{t}\right|} \neq\left|X_{t}\right|\right\}\right)=I_{1}+I_{2} .
$$

Using the Wald identity, we have

$$
I_{2}=\mathbb{E}_{A}\left(\max _{1 \leqslant t \leqslant \tau_{A}} 1_{\left\{\left|X_{t}\right|>x\right\}}\right) \leqslant \mathbb{E}_{A}\left(\sum_{t=1}^{\tau_{A}} 1_{\left\{\left|X_{t}\right|>x\right\}}\right)=\mathbb{E}_{A}\left(\tau_{A}\right) \mathbb{P}(|X|>x) .
$$

Write $k_{0}=\lceil\alpha\rceil$ and choose $0<\beta<1$ such that $\beta k_{0}>\alpha$. Since $k_{0}\left(k_{0}-1\right) \geqslant \alpha(\alpha-1)$ for $\alpha>1$, we will choose $\beta$ such that $p / \beta=k_{0}-1$. Markov's inequality yields

$$
I_{1} \leqslant c \frac{\mathbb{E}_{A}\left(\sum_{t=1}^{\tau_{A}} \overline{\left|X_{t}\right|}\right)^{\beta k_{0}}}{x^{\beta k_{0}}} \leqslant c \frac{\mathbb{E}_{A}\left(\sum_{t=1}^{\tau_{A}} \overline{\left|X_{t}\right|}\right)^{k_{0}}}{x^{\beta k_{0}}}
$$


Note that $\left(\overline{\mid X}_{t}{ }^{\beta}\right)$ satisfies $\left(\mathbf{D C}_{k_{0}-1}\right)$. Under the latter condition we may apply Proposition 4.7 of [40. to get $\mathbb{E}_{A}\left(\sum_{t=1}^{\tau_{A}}{\overline{\left|X_{t}\right|}}^{\beta}\right)^{k_{0} \leqslant c \mathbb{E}|X|}{ }^{\beta k_{0}}$. An application of Karamata's theorem shows that the right-hand side is bounded by $c \mathbb{P}(|X|>x)$. This concludes the proof of (7.1).

Now we turn to the proof of (7.2). Abusing notation, we write $\bar{X}=\mathbb{1}_{\{|X| \leqslant x \delta\}}$ for any fixed $\delta$. Then

$$
\mathbb{P}\left(\sum_{t=1}^{\tau_{A}}\left|X_{t}\right|>x, \tau_{A} \leqslant n\right) \leqslant \mathbb{P}\left(\sum_{t=1}^{\tau_{A}} \overline{\left|X_{t}\right|}>x / 2, \tau_{A} \leqslant n\right)+\mathbb{P}\left(\cup_{t=1}^{\tau_{A}}\left\{\overline{\left|X_{t}\right|} \neq\left|X_{t}\right|\right\}\right)=I_{1}^{\prime}+I_{2}^{\prime} .
$$

Since $\mathbb{E} \tau_{A}<\infty$ and $X$ is regularly varying, we have

$$
I_{2}^{\prime} \leqslant \mathbb{E}\left(\tau_{A}\right) \mathbb{P}(|X|>x \delta)=o(n \mathbb{P}(|X|>x)) .
$$

Similar arguments as above yield

$$
I_{1}^{\prime} \leqslant c \frac{\mathbb{E}\left(\sum_{t=1}^{\tau_{A}} \overline{\left|X_{t}\right|} \mathbb{1}_{\left\{\tau_{A} \leqslant n\right\}}\right)^{\beta k_{0}}}{x^{\beta k_{0}}} \leqslant c \frac{\mathbb{E}\left(\sum_{t=1}^{n} \overline{\left|X_{t}\right|} \mathbb{1}_{\left\{\tau_{A} \geqslant t\right\}}\right)^{\beta k_{0}}}{x^{\beta k_{0}}} .
$$

An argument similar to the one used in the proof of Theorem 4.6 in [40] shows that

$$
\mathbb{E}\left(\sum_{t=1}^{n}{\overline{\left|X_{t}\right|}}^{\beta} \mathbb{1}_{\left\{\tau_{A} \geqslant t\right\}}\right)^{k_{0}} \leqslant c \mathbb{E}\left(\sum_{t=1}^{n}{\overline{\left|X_{t}\right|}}^{\beta k_{0}} \mathbb{1}_{\left\{\tau_{A} \geqslant t\right\}}\right) .
$$

Finally, an application of Pitman's identity [45], Proposition 4.7 in [40] and Karamata's theorem yield

$$
\begin{aligned}
\mathbb{E}\left(\sum_{t=1}^{n}{\overline{\mid X_{t}}}^{\beta k_{0}} \mathbb{1}_{\left\{\tau_{A} \geqslant t\right\}}\right) & =\mathbb{P}\left(X_{0} \in A\right) \mathbb{E}_{A}\left(\sum_{k=0}^{\tau_{A}-1} \sum_{t=1}^{n}{\overline{\left|X_{k+t}\right|}}^{\beta k_{0}} \mathbb{1}_{\left\{\tau_{A} \geqslant k+t\right\}}\right) \\
& \leqslant n \mathbb{P}\left(X_{0} \in A\right) \mathbb{E}_{A}\left(\sum_{t=1}^{\tau_{A}} \overline{\left|X_{t}\right|} \bar{k}^{\beta k_{0}}\right) \\
& \leqslant c n \mathbb{E} \mid \overline{|X|}{ }^{\beta k_{0}} \sim c n x^{\beta k_{0}} \delta^{\beta k_{0}-\alpha} \mathbb{P}(|X|>x) .
\end{aligned}
$$

Since $\beta k_{0}>\alpha$ and we can make $\delta$ as small as we like, we conclude that $I_{1}^{\prime}=o(n(\mathbb{P}(|X|>x))$. This concludes the proof of $(7.2)$.

Now we are ready to prove part (1). Since $\tau_{A}$ has exponential moment, it follows that $\mathbb{P}\left(\tau_{A}>\right.$ $n)=o\left(\mathbb{P}\left(|X|>\lambda_{n}\right)\right.$. Therefore we may prove the result on the event $\left\{\tau_{A} \leqslant n\right\}$. We write for simplicity $\mathbb{P}_{n}(\cdot)=\mathbb{P}\left(\cdot \cap\left\{\tau_{A} \leqslant n\right\}\right)$. In view of Lemma 7.1 and the decomposition (6.2) of $S_{n}$ we may neglect the sums over the first and last cycles and it suffices to prove the large deviation principle for the process $\sum_{t=1}^{N_{A}(n)} S(t)$ over independent cycles. Observe that

$$
\frac{\mathbb{P}_{n}\left(\lambda_{n}^{-1} \sum_{t=1}^{N_{A}(n)} S(t) \in \cdot\right)}{n \mathbb{P}\left(|X| \geqslant \lambda_{n}\right)}=\frac{\mathbb{P}_{n}\left(\lambda_{n}^{-1} \sum_{t=1}^{N_{A}(n)} S(t) \in \cdot\right)}{n \mathbb{P}\left(|S(1)| \geqslant \lambda_{n}\right)} \frac{\mathbb{P}\left(|S(1)| \geqslant \lambda_{n}\right)}{\mathbb{P}\left(|X| \geqslant \lambda_{n}\right)} .
$$

The same arguments as in the proof of Lemma 4.12 in 40 (here the conditions $\lambda_{n} \rightarrow \infty$ and $\lambda_{n} / n^{\delta+1 / \alpha} \rightarrow \infty$ for some $\delta>0$ are crucial) show that for any small $\xi, \varepsilon>0$, and any set $B$ bounded away from zero,

$$
\begin{aligned}
\frac{(1-\varepsilon) \mathbb{P}\left(\lambda_{n}^{-1}(1+\xi)^{-1}(1+\varepsilon)^{-1} S(1) \in B\right)}{\mathbb{E}\left(\tau_{A}\right) \mathbb{P}\left(|S(1)|>\lambda_{n}\right)} & \leqslant \frac{\mathbb{P}_{n}\left(\lambda_{n}^{-1} \sum_{t=1}^{N_{A}(n)} S(t) \in B\right)}{n \mathbb{P}\left(|S(1)|>\lambda_{n}\right)}+o(1) \\
& \leqslant \frac{\mathbb{P}\left(\lambda_{n}^{-1}(1-\xi)^{-1} S(1) \in B\right)}{\mathbb{E}\left(\tau_{A}\right) \mathbb{P}\left(|S(1)|>\lambda_{n}\right)}+o(1) .
\end{aligned}
$$


Assume first that the cluster index $b$ does not vanish. In view of part (2) of Theorem 4.1 we know that $S(1)$ is regularly varying with index $\alpha$ and spectral measure $\mathbb{P}_{\Theta^{\prime}}$ given by (4.3), and we also know that

$$
\frac{\mathbb{P}\left(\lambda_{n}^{-1} S(1) \in \cdot\right)}{\mathbb{P}\left(|S(1)|>\lambda_{n}\right)} \stackrel{v}{\rightarrow} \mu_{S(1)}(\cdot),
$$

for a non-null Radon measure $\mu_{S(1)}$. Hence, letting $\varepsilon \rightarrow 0$ and $\xi \rightarrow 0$, we conclude that

$$
\frac{\mathbb{P}_{n}\left(\lambda_{n}^{-1} \sum_{t=1}^{N_{A}(n)} S(t) \in \cdot\right)}{n \mathbb{P}\left(|S(1)|>\lambda_{n}\right)} \stackrel{v}{\rightarrow} \frac{\mu_{S(1)}(\cdot)}{\mathbb{E}\left(\tau_{A}\right)} .
$$

It remains to determine the limit of $\mathbb{P}(|S(1)|>x) / \mathbb{P}(|X|>x)$ as $x \rightarrow \infty$. By virtue of the proof of Theorem 4.1, $a_{n}^{-1} \sum_{t=1}^{n / \mathbb{E} \tau_{A}} S(t) \stackrel{d}{\rightarrow} \xi_{\alpha}$. Then necessarily

$$
\frac{n}{\mathbb{E} \tau_{A}} \mathbb{P}\left(a_{n}^{-1} S(1) \in \cdot\right) \stackrel{v}{\rightarrow} \nu_{\alpha}(\cdot),
$$

where $\nu_{\alpha}$ is the Lévy measure of $\xi_{\alpha}$. Hence

$$
\frac{\mathbb{P}\left(|S(1)|>a_{n}\right)}{\mathbb{P}\left(|X|>a_{n}\right)} \sim n \mathbb{P}\left(|S(1)| \geqslant a_{n}\right) \rightarrow \mathbb{E} \tau_{A} \Gamma_{\alpha}\left(\mathbb{S}^{d-1}\right),
$$

where $\Gamma_{\alpha}$ is the spectral measure of $\nu_{\alpha}$. But from part (2) of Theorem 4.1 we know that $n \mathbb{P}(|S(1)| \geqslant$ $\left.a_{n}\right) \rightarrow \mathbb{E} \tau_{A} \int_{\mathbb{S}^{d-1}} b(\theta) d P_{\Theta}(\theta)$. This proves the result in the non-generate case $b \neq 0$.

In the degenerate case $b=0, \mathbb{P}(|S(1)| \geqslant x)=o(\mathbb{P}(|X| \geqslant x))$ as $x \rightarrow \infty$. By independence of the cycles and since $\lambda_{n} / a_{n} \rightarrow \infty$, for any set $B$ bounded away from zero, some $\gamma>0$,

$\mathbb{P}_{n}\left(\lambda_{n}^{-1} \sum_{t=1}^{N_{A}(n)} S(t) \in B\right) \leqslant \mathbb{P}\left(\left|\sum_{t=1}^{N_{A}(n)} S(t)\right|>\gamma \lambda_{n}\right) \leqslant n c \mathbb{P}\left(|S(1)| \geqslant c \lambda_{n}\right)=o\left(n \mathbb{P}\left(|X|>a_{n}\right)\right)=o(1)$.

The desired result in the degenerate case follows.

7.2. Proof of part (2): The case $\alpha>2$. We only consider the non-degenerate case $b \neq 0$. We will apply Theorem 4.6 in [40] for functions of Markov chains in the case $d=1$.

Theorem 7.2. Let $\left(G_{t}\right)=\left(f\left(\Phi_{t}\right)\right)$ be a 1-dimensional functional of a strictly stationary $\mathbb{R}$-valued irreducible aperiodic Markov chain $\left(\Phi_{t}\right)$. Write $S_{n}(G)=G_{1}+\cdots+G_{n}, n \geqslant 1$, for the corresponding random walk. Assume that the following conditions are satisfied.

(1) The regular variation condition $\left(\mathbf{R V}_{\alpha}\right)$ for some $\alpha>2$ and $\mathbb{E} G=0$.

(2) The anti-clustering condition $(\mathbf{A C})_{\alpha}$ :

$$
\lim _{k \rightarrow \infty} \limsup _{n \rightarrow \infty} \sup _{x \in \Lambda_{n}} \delta_{k}^{-\alpha} \sum_{j=k}^{n} \mathbb{P}\left(\left|G_{j}\right|>x \delta_{k}|| G_{0} \mid>x \delta_{k}\right)=0 .
$$

for a sequence $\delta_{k}=o\left(k^{-2}\right), k \rightarrow \infty$, and sets $\left(\Lambda_{n}\right)$ such that $b_{n}=\inf \Lambda_{n} \rightarrow \infty$ as $n \rightarrow \infty$.

(3) The limit $b_{+}=\lim _{k \rightarrow \infty}\left(b_{+}(k+1)-b_{+}(k)\right)$ exists, where the constants $\left(b_{+}(k)\right)$ are defined in Theorem 6.1.

(4) The drift condition $\left(\mathbf{D C}_{p}\right)$ for every $p<\alpha$.

Then the precise large deviation principle

$$
\lim _{n \rightarrow \infty} \sup _{x \in \Lambda_{n}}\left|\frac{\mathbb{P}\left(S_{n}(G)>x\right)}{n \mathbb{P}(|G|>x)}-b_{+}\right|=0,
$$


holds if $\Lambda=\left(b_{n}, c_{n}\right)$ for any sequence $\left(b_{n}\right)$ satisfying $b_{n}=n^{0.5+\varepsilon}$ for any $\varepsilon>0$, and $\left(c_{n}\right)$ such that $c_{n}>b_{n}$ and

$$
\mathbb{P}\left(\tau_{A}>n\right)=o\left(n \mathbb{P}\left(|G|>c_{n}\right)\right),
$$

where $\tau_{A}=\tau_{A}(1)$ is the first hitting time of the atom A of the Markov chain; see Section 2.2.

We will apply this result to $G_{t}=\theta^{\prime} X_{t}, t \in \mathbb{Z}$, for any fixed $\theta \in \mathbb{S}^{d-1}$ with $b(\theta) \neq 0$. Note that (7.4) is satisfied since $\tau_{A}$ has exponential moment. Condition $\left(\mathbf{R V}_{\alpha}\right)$ for $\left(G_{t}\right)$ is satisfied by regular variation of $\left(X_{t}\right)$ in all non-degenerate cases $b(\theta) \neq 0$. The existence of the limits $b_{+}=\lim _{k \rightarrow \infty}\left(b_{+}(k+1)-b_{+}(k)\right)=b(\theta) / \mathbb{E}\left|\theta^{\prime} \Theta_{0}\right|^{\alpha}$ (here we assume that $\mathbb{E}\left|\theta^{\prime} \Theta_{0}\right|^{\alpha} \neq 0$ ) is ensured by Theorem 3.2. It remains to check condition $(\mathbf{A C})_{\alpha}$ for $\left(G_{t}\right)$ under $\left(\mathbf{D C}_{p}\right)$ for $\left(G_{t}\right)$ for every $p<\alpha$. Note that $\left(\mathbf{D C}_{p}\right)$ for $\left(X_{t}\right)$ implies $\left(\mathbf{D C}_{p}\right)$ for $\left(G_{t}\right)$. Using Markov's inequality of order $p<\alpha$ and (6.1), we obtain the following bound for $k \geqslant 1, x \in \Lambda_{n}$ :

$$
\begin{aligned}
\sum_{j=k}^{n} \mathbb{P}\left(\left|G_{j}\right|>x \delta_{k}|| G_{0} \mid>x \delta_{k}\right) & \leqslant \sum_{j=k}^{n} \frac{\mathbb{E}\left(\left|G_{j}\right|^{p} \mathbb{1}_{\left\{\left|G_{0}\right|>x \delta_{k}\right\}}\right)}{x^{p} \delta_{k}^{p} \mathbb{P}\left(\left|G_{0}\right|>x \delta_{k}\right)} \\
& \leqslant \sum_{j=k}^{n}\left(\frac{\beta^{j-1} \mathbb{E}\left(\left|X_{0}\right|^{p} \mathbb{1}_{\left\{\left|X_{0}\right|>x \delta_{k}\right\}}\right)}{x^{p} \delta_{k}^{p} \mathbb{P}\left(\left|G_{0}\right|>x \delta_{k}\right)}+\frac{c}{x^{p} \delta_{k}^{p}}\right) \\
& \leqslant c\left(\frac{\beta^{k} \mathbb{E}\left(\left|X_{0}\right|^{p} \mathbb{1}_{\left\{\left|X_{0}\right|>x \delta_{k}\right\}}\right)}{x^{p} \delta_{k}^{p} \mathbb{P}\left(\left|X_{0}\right|>x \delta_{k}\right)}+\frac{n}{x^{p} \delta_{k}^{p}}\right) .
\end{aligned}
$$

The second term is of the order $O\left(n b_{n}^{-p}\right)=o(1)$ uniformly for $x \in \Lambda_{n}$ since $p$ can be chosen larger than 2 such that $p(0.5+\varepsilon)>1$. The first term converges to $c \beta^{k}$ as $n \rightarrow \infty$ uniformly for $x \in \Lambda_{n}$, by applications of Karamata's Theorem and the uniform convergence theorem of regular variation. We conclude that $(\mathbf{A C})_{\alpha}$ holds as $\delta_{k}^{-1} \beta^{k} \rightarrow 0$ as $k \rightarrow \infty$ if we choose $\delta_{k}=k^{-2-\varepsilon^{\prime}}$ for $\varepsilon^{\prime}>0$ sufficiently small. Thus all conditions of Theorem 7.2 are satisfied for $\left(G_{t}\right)=\left(\theta^{\prime} X_{t}\right)$ and therefore (7.3) applies. Since $\mathbb{P}\left(\left|\theta^{\prime} X\right|>x\right) / \mathbb{P}(|X|>x) \rightarrow \mathbb{E}\left[\left|\theta^{\prime} X\right|^{\alpha}\right]$ we can also write (7.3) in the form (4.3).

Now choose $\left(\lambda_{n}\right)$ as in the formulation of the theorem and apply Lemma A.1 below. This proves the theorem.

\section{Appendix A.}

The following result is useful for proving multivariate large deviation results and central limit theorems.

Lemma A.1. Assume that $\left(X_{t}\right)$ is an $\mathbb{R}^{d}$-valued strictly stationary sequence which is regularly varying with index $\alpha>0$ and satisfies the one-dimensional large deviation principle

$$
\frac{\mathbb{P}\left(\theta^{\prime} S_{n}>\lambda_{n}\right)}{n \mathbb{P}\left(|X|>\lambda_{n}\right)} \rightarrow b(\theta), \quad \theta \in \mathbb{S}^{d-1},
$$

for some sequence $\lambda_{n} \rightarrow \infty$ such that $n \mathbb{P}\left(|X|>\lambda_{n}\right) \rightarrow 0$. Moreover, assume that $\alpha \notin \mathbb{N}$ or $b(\cdot)=b(-\cdot)$. Then (4.1) holds.

Proof. Define the measures

$$
m_{n}(\cdot)=\frac{\mathbb{P}\left(\lambda_{n}^{-1} S_{n} \in \cdot\right)}{n \mathbb{P}\left(|X|>\lambda_{n}\right)}, \quad n \geqslant 1,
$$

on the Borel $\sigma$-field of $\overline{\mathbb{R}}_{0}^{d}$. We conclude from th.1 that for any Borel set $B$ bounded away from zero,

$$
\sup _{n \geqslant 1} m_{n}(B)<\infty
$$


This means that $\left(m_{n}(B)\right)$ is vaguely tight; see [31, 46. In view of A.1), any vague subsequential limit $\mu$ of $\left(m_{n}\right)$ satisfies the relation (4.2). For non-integer $\alpha$, the latter property combined with the proof of Theorem 1.1 in $\left[3\right.$ shows that all vague subsequential limits of $\left(m_{n}\right)$ are identical and uniquely determined by the property (4.2). Hence (4.1) holds and the limit $\nu_{\alpha}$ is given by (4.2). A careful study of the proof of Theorem 1.1 in 3 shows that the proof remains valid if the subsequential limits have the property $\mu(\cdot)=\mu(-\cdot)$ which follows if $b(\cdot)=b(-\cdot)$.

Acknowledgments. We would like to thank the referee for careful reading of our paper and for useful commments.

\section{REFERENCES}

[1] Alsmeyer, G. And Mentemeier, S. (2012) Tail behavior of stationary solutions of random difference equations: the case of regular matrices. J. Diff. Equation Appl. 18, 1305-1332.

[2] Bartkiewicz, K., Jakubowski, A., Mikosch, T. and Wintenberger, O. (2011) Stable limits for sums of dependent infinite variance random variables. Probab. Th. Rel. Fields 150, 337-372.

[3] Basrak, B., Davis, R.A. And Mikosch. T. (2002) A characterization of multivariate regular variation. Ann. Appl. Probab. 12, 908-920.

[4] Basrak, B., Davis, R.A. and Mikosch. T. (2002) Regular variation of GARCH processes. Stoch. Proc. Appl. 99, 95-116.

[5] Basrak, B., Krizmanić, D. and Segers, J. (2012) A functional limit theorem for dependent sequences with infinite variance stable limits. Ann. Probab. To appear.

[6] Basrak, B. And Segers, J. (2009) Regularly varying multivariate time series. Stoch. Proc. Appl. 119, 1055-1080.

[7] Bingham, N.H., Goldie, C.M. And Teugels, J.L. (1987) Regular Variation. Cambridge University Press, Cambridge.

[8] Boman, J. And Lindskog, F. (2009) Support theorems for the Radon transform and Cramér-Wold theorems. $J$. Theor. Probab. 22, 683-710.

[9] Bougerol, P. and Picard, N. (1992) Strict stationarity of generalized autoregressive processes. Ann. Probab. 20, 1714-1730.

[10] Breiman, L. (1965) On some limit theorems similar to the arc-sin law. Theory Probab. Appl. 10, 323-331.

[11] Brockwell, P.J. and Davis, R.A. (1991) Time Series: Theory and Methods, 2nd edition Springer-Verlag, New York.

[12] Buraczewski, D., Damek, E. and Guivarc'H, Y. (2012) Convergence to stable laws for a class of multidimenisional stochastic recursions. Probab. Th. Rel. Fields. To appear.

[13] Buraczewski, D., Damek, E., Guivarc'h, Y., Hulanicki, A. and Urban, R. (2012) Tail homogeneity of stationary measures for some multidimensional stochastic recursions. Preprint.

[14] Buraczewski, D., Damek, E., Mikosch, T. And Zienkiewicz, J. (2013) Large deviations for solutions to stochastic recurrence equations under Kesten's condition. Ann. Probab., to appear.

[15] Damek, E., Mentemeier, S., Mirek, M. and Zienkiewicz, J. (2011) Convergence to stable laws for multidimensional stochastic recursions: the case of regular matrices. Preprint.

[16] Davis, R.A. And Hsing, T. (1995) Point process and partial sum convergence for weakly dependent random variables with infinite variance. Ann. Prob. 23, 879-917.

[17] Davis, R.A. And Mikosch, T. (1998) Limit theory for the sample ACF of stationary process with heavy tails with applications to ARCH. Ann. Statist. 26, 2049-2080.

[18] Doukhan, P. And Louhichi, S. (1999) A new weak dependence condition and applications to moment inequalities. Stochastic Process. Appl. 84, 313-342.

[19] Feller, W. (1971) An Introduction to Probability Theory and Its Applications. Vol. II. Second edition. Wiley, New York.

[20] Gao, Z., Guivarc'h and Y. Le Page, E. (2011) Spectral gap properties and convergence to stable laws for affine random walks on $\mathbb{R}^{d}$. Preprint.

[21] Gimman, I.I. and Skorohod, A.V. (1974) The Theory of Stochastic Processes I. Springer, Berlin.

[22] GuivarC'H, Y. (2006) Heavy Tail Properties of Stationary Solutions of Multidimensional Stochastic Recursions. IMS Lecture Notes Monogr. 48, Beachwood, OH.

[23] Gut, A. (1988) Stopped Random Walks. Springer, New York. 
[24] Hult, H. and Lindskog, F. (2005) Extremal behavior of regularly varying stochastic processes. Stoch. Proc. Appl. 115, 249-274.

[25] Hult, H. And Lindskog, F. (2006) Regular variation for measures on metric spaces. Publ. Inst. Math. (Beograd) (N.S.) 80(94), 121-140,

[26] Hult, H. And Lindskog, F. (2006) On Kesten's counterexample to the Cramŕ-Wold device for regular variation. Bernoulli 12, 133-142.

[27] Hult, H., Lindskog, F., Mikosch, T. and Samorodnitsky, G. (2005) Functional large deviations for multivariate regularly varying random walks. Ann. Appl. Probab. 15, 2651-2680.

[28] Jakubowski, A. (1993) Minimal conditions in p-stable limit theorems. Stoch. Proc. Appl. 44, 291-327.

[29] Jakubowski, A. (1997) Minimal conditions in p-stable limit theorems - II. Stoch. Proc. Appl. 68, 1-20.

[30] Jessen, A.H. and Mikosch, T. (2006) Regularly varying functions. Publ. Inst. Math. Nouvelle Série 80(94), $171-192$.

[31] Kallenberg, O. (1983) Random Measures, 3rd edition. Akademie-Verlag, Berlin.

[32] Kesten, H. (1973) Random difference equations and renewal theory for products of random matrices. Acta Math. 131, 207-248.

[33] Klüppelberg, C. and Pergamenchtchikov, S. (2007) Extremal behaviour of models with multivariate random recurrence representation. Stoch. Proc. Appl. 117, 432-456.

[34] Konstantinides, D. and Mikosch, T. (2004) Large deviations and ruin probabilities for solutions to stochastic recurrence equations with heavy-tailed innovations. Ann. Probab. 33, 1992-2035.

[35] Leadbetter, M.R., Lindgren, G. and Rootzén, H. (1983) Extremes and Related Properties of Random Sequences and Processes. Springer, Berlin.

[36] Meyn, S.P. And Tweedie, R.L. (1993), Markov Chains and Stochastic Stability. Springer, London.

[37] Mikosch, T. And StĂRicĂ, C. (2000) Limit theory for the sample autocorrelations and extremes of a GARCH $(1,1)$ process. Ann. Statist. 28, 1427-1451.

[38] Mikosch, T. and Starica, C. (2003) Long-range dependence effects and ARCH modeling. In: Doukhan, P., Oppenheim, G. and Taqqu, M.S. (Eds.) Long-Range Dependence. Birkhäuser, Boston, pp.439-459.

[39] Mikosch, T. and SAmorodnitsky, G. (2000) The supremum of a negative drift random walk with dependent heavy-tailed steps. Ann. Appl. Probab. 10, 1025-1064.

[40] Mikosch, T. and Wintenberger, O. (2013) Precise large deviations for dependent regularly varying sequences. Probab. Rel. Fields, to appear.

[41] Nagaev, A.V. (1969) Integral limit theorems for large deviations when Cramér's condition is not fulfilled I,II. Theory Probab. Appl. 14, 51-64 and 193-208.

[42] Nagaev, S.V. (1979) Large deviations of sums of independent random variables. Ann. Probab. 7, 745-789.

[43] Nummelin, E. (1984) General Irreducible Markov Chains and Non-Negative Operators. Cambridge University Press, Cambridge.

[44] Petrov, V.V. (1995) Limit Theorems of Probability Theory. Oxford University Press, Oxford (UK).

[45] Pitman, J. (1977) Occupation measures for Markov chains. Adv. Appl. Probab. 9, 69-86.

[46] Resnick, S.I. (1987) Extreme Values, Regular Variation, and Point Processes. Springer, New York.

[47] Resnick, S.I. (2007) Heavy-Tail Phenomena: Probabilistic and Statistical Modeling. Springer, New York.

[48] Resnick, S.I. And Willekens, E. (1991) Moving averages with random coefficients and random coefficient autoregressive models. Stoch. Models 7, 511-525.

[49] Rvaceva, E.L. (1962) On domains of attraction of multi-dimensional distributions. Select. Transl. Math. Statist. and Probability of the AMS 2. 183-205.

[50] Samorodnitsky, G. And Taqqu, M.S. (1994) Stable Non-Gaussian Random Processes. Chapman \& Hall, New York.

[51] Samur, J.D. (2004) A regularity condition and a limit theorem for Harris ergodic Markov chains, Stoch. Proc. Appli., 111, 207-235.

[52] Tyran-Kamińska, M. (2010) Convergence to Lévy stable processes under some weak dependence conditions. Stoch. Proc. Appl. 120 (2010), 1629-1650.

Thomas Mikosch, University of Copenhagen, Department of Mathematics, Universitetsparken 5, DK2100 Copenhagen, Denmark

E-mail address: mikosch@math.ku.dk

Olivier Wintenberger, Université de Paris-Dauphine and CREST-LFA, Centre De Recherche en Mathématiques de la Décision UMR CNRS 7534, Place du Maréchal De Lattre De Tassigny, 75775 Paris Cedex 16, France

E-mail address: owintenb@ceremade.dauphine.fr 